C-A/AP/\#366

August 2010

\title{
High Precision Tune and Coupling Measurements and Tune/Coupling Feedback in RHIC
}

M. Minty, A. Curcio, C. Dawson, C. Degen, Y. Luo, G. Marr, B. Martin, A. Marusic, K. Mernick, P. Oddo, T. Russo, V. Schoefer, R. Schroeder, C. Schultheiss, M. Wilinski

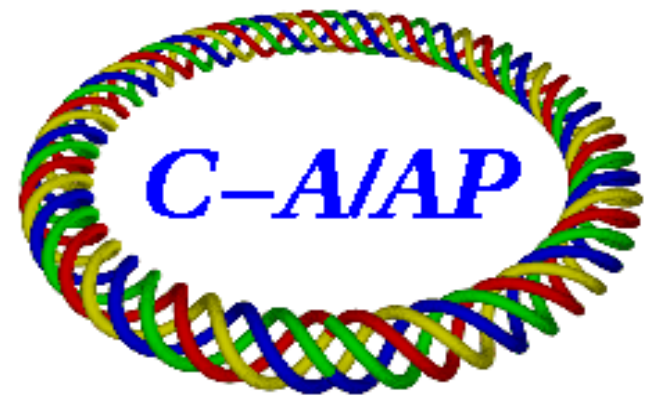

\section{Collider-Accelerator Department Brookhaven National Laboratory Upton, NY 11973}

Notice: This document has been authorized by employees of Brookhaven Science Associates, LLC under Contract No. DE-AC02-98CH10886 with the U.S. Department of Energy. The United States Government retains a nonexclusive, paid-up, irrevocable, world-wide license to publish or reproduce the published form of this document, or allow others to do so, for United States Government purposes. 


\title{
High Precision Tune and Coupling Measurements and Tune/Coupling Feedback in RHIC
}

\author{
M. Minty, A. Curcio, C. Dawson, C. Degen, Y. Luo, G. Marr, B. Martin, \\ A. Marusic, K. Mernick, P. Oddo, T. Russo, V. Schoefer, \\ R. Schroeder, C. Schultheiss, M. Wilinski \\ Brookhaven National Laboratory, Upton, NY 11973, USA
}

\begin{abstract}
Precision measurement and control of the betatron tunes and betatron coupling in RHIC are required for establishing and maintaining both good operating conditions and, particularly during the ramp to high beam energies, high proton beam polarization. While the proof-of-principle for simultaneous tune and coupling feedback was successfully demonstrated earlier, routine application of these systems has only become possible recently. Following numerous modifications for improved measurement resolution and feedback control, the time required to establish full-energy beams with the betatron tunes and coupling regulated by feedback was reduced from several weeks to a few hours. A summary of these improvements, select measurements benefitting from the improved resolution and a review of system performance are the subject of this report.
\end{abstract}

\section{Introduction}

The successful demonstration of simultaneous tune and coupling feedback in RHIC [1] during Run-6 was realized using direct-diode detection [2] for precision tune measurements (also referred to as BBQ, an acronym for base-band Q, or tune, measurements) and eigenmode projection [3-5] for direct measurement and hence control of the eigenmodes of the betatron oscillations. Since these very successful tests, routine use of tune and coupling feedback has been hampered by interferences from mains harmonics and complications with near-zero chromaticity [6], from rich spectral content at injection energy when accelerating ion beams [6,7], and from practicalities such as loss of lock of the phase lock loop used in determining the betatron frequencies [7,8]. Prior to and during initial system commissioning for Run-9, numerous hardware and software modifications were implemented which significantly improved the measurement resolution and eliminated multiple sources of systematic errors. As a result, all the above difficulties have been overcome.

In addition to establishing for the first time routine implementation of tune and coupling feedback, the improved measurement resolution has allowed for detection and characterization of several interesting phenomena including significant tune modulations experienced by both beams in both planes of appreciable magnitude compared to the total beam-beam parameter. High-resolution beam transfer function measurements revealed not only strong coherent modes induced by the beam-beam interaction but also a clear measure of significant structure between these modes.

In this report a brief review of the technique used for measurement and control of the tunes and betatron coupling is given in section 2. In section 3 the achieved resolution of the tune measurements is evaluated and experimental data are presented showing practical limitations arising from externally driven tune modulations. In section 4 experimental data are shown demonstrating the improved precision in measuring and controlling global coupling. In section 5 illustrative examples are given as characterizing the performance of the tune/coupling feedback systems in Run-9 with application chronologies given in Appendix A and a post-run performance analysis in Appendix B. In section 6 measurements of the transverse beam transfer functions are presented. Sections 7 and 8 summarize, respectively, the hardware and software improvements without which high precision tune and coupling measurements and consequently routine tune/coupling feedback or operation of RHIC at near-integer tunes could not have been possible. A summary and outlook are given in section 9 . 


\section{Overview of Tune/Coupling Measurement and Control Technique}

In the following we describe the technique used to measure and feedback on the betatron tunes and betatron coupling. We refer the reader to Ref. [1] for more detail on the experimental hardware used for the tune measurements. Attention here is primarily devoted to the data processing algorithms which have not been previously presented in detail and for which numerous modifications during Run-9 led to the significantly improved precision and robustness of the tune and coupling feedback systems.

A conceptual sketch illustrating system functionality for tune measurement and feedback in one transverse plane in the absence of betatron coupling is given in Fig. 1. The transverse position (horizontal denoted by $\mathrm{x}$, vertical by y) of the beam is detected using a 1 meter long dual-plane stripline detector. The beaminduced signals are brought out of the tunnel through long cables to a support building housing the electronics for signal processing. For a given plane, the signals from the two corresponding striplines pass through diodes at the input of the analog front end (AFE) module within which the difference signal is generated followed by various filters with a net passband of $\sim 25 \mathrm{kHz}$ around the expected betatron frequency, or in the implementation at RHIC, around the aliased betatron frequency. As an example, for Run-9 with fractional betatron tunes of $Q=0.68$ and a revolution frequency $\mathrm{f}_{\text {rev }}=78 \mathrm{kHz}$, the aliased betatron frequency is $\mathrm{f}_{\beta}=(1-\mathrm{Q}) * \mathrm{f}_{\text {rev }} \sim 25 \mathrm{kHz}$. This combination of so-called direct diode detection and AFE composes the basis of what is widely referred to as base-band tune (BBQ) measurements [2].

The AFE output signal is input to an analog to digital converter (ADC) sampled nominally at four times the betatron frequency. The AFE output is therefore sampled at "in-phase" values (denoted by I) and "quadrature"-values (those separated in phase by $\frac{\pi}{2}$, denoted by Q) by this 4-point-per-period I/Q demodulator. The detected I and Q values acquired over 8 periods are passed through a finite impulse response (FIR) filter after which the amplitude, $\mathrm{A}=\sqrt{\langle Q\rangle^{2}+\langle I\rangle^{2}}$, and phase, $\phi=\operatorname{atan}\left(\frac{\mathrm{Q}}{\mathrm{I}}\right)$, are computed. Added to this phase are a user-adjustable offset $\phi_{0}$ to compensate for static phase shifts in the system and a frequency-dependent phase correction $(\mathrm{d} \phi / \mathrm{df}) \Delta \mathrm{f}$, where $(\mathrm{d} \phi / \mathrm{df})$ represents the measured phase dependence of the AFE and $\Delta \mathrm{f}$ is the frequency deviation from reference as required during the energy ramp (or in measurements of the beam transfer functions). Trigonometric projection of the rotated vector given by $\mathrm{A}$ and the net phase $\phi+\phi_{0}+(\mathrm{d} \phi / \mathrm{df}) \Delta \mathrm{f}$ determine new I and Q values denoted by $\widetilde{\mathrm{I}}$ and $\tilde{\mathrm{Q}}$ where these now represent sampling near the peaks and zeros of the incident AFE output signal, respectively.

The normalized quadrature-value, $\tilde{\mathrm{Q}} / \tilde{\mathrm{A}}$ with $\tilde{\mathrm{A}}^{2}=\sqrt{\tilde{\mathrm{Q}}^{2}+\tilde{\mathrm{I}}^{2}}$, is then input to a proportional-integralderivative (PID) loop which, for Run-9 as in past runs, was used without the derivative component so comprising a PI loop. The loop output is the fractional betatron tune $\mathrm{Q}_{\beta}$ given by the nominal tune, $\mathrm{Q}_{\beta}{ }^{\text {nom }}$, plus the correction which is proportional to the phase error between the desired and measured betatron frequencies. The NCO takes as input the factor $N=\left(2^{32} / h\right) Q_{\beta}$, where $h$ is the harmonic number given by the ratio of the rf frequency to the revolution frequency. For beam excitation at the betatron frequency, the NCO output is filtered, amplified by a user-selectable amount, and used as input to a $1 \mathrm{~m}$ stripline kicker.

Measurements of the beam transfer functions use the same setup of experimental hardware and data processing as shown in Fig. 1 with two exceptions: the tune tracking loop is opened (denoted conceptually by a switch to ground beneath the NCO in Fig. 1) and the NCO is programmed to vary the frequency over a user-specified range in discrete steps. Once the frequency scan is initiated, the NCO is set to a fixed frequency, samples are acquired and logged, and after the given dwell time, the NCO frequency is stepped incrementally to the next value.

Tune feedback adjusts the main quadrupole (both focusing and defocusing) magnet currents to keep the betatron tunes equal to the desired values. As input are used the difference between the measured betatron frequency $\mathrm{Q}_{\beta}$ averaged over 16 samples, $<\mathrm{Q}_{\beta}>$, and the desired nominal value $\mathrm{Q}_{\beta}{ }^{\text {nom }}$, shown as $\Delta \mathrm{Q}_{\beta \mathrm{x}}$ in Fig. 1, together with the data obtained in the orthogonal plane, $\Delta \mathrm{Q}_{\beta \mathrm{y}}$ (shown entering from the lower right of Fig. 1). These error signals are converted to energy-independent field strength corrections using a twoby-two matrix of elements specifying the derivatives of the required field strengths per change in betatron tune, as given by the accelerator optical model. The correction strengths are then added to the nominal 
operating strengths and then converted to currents for the power supplies powering the quadrupole magnets. The closed-loop response of the tune feedback loop is regulated using PI loops.

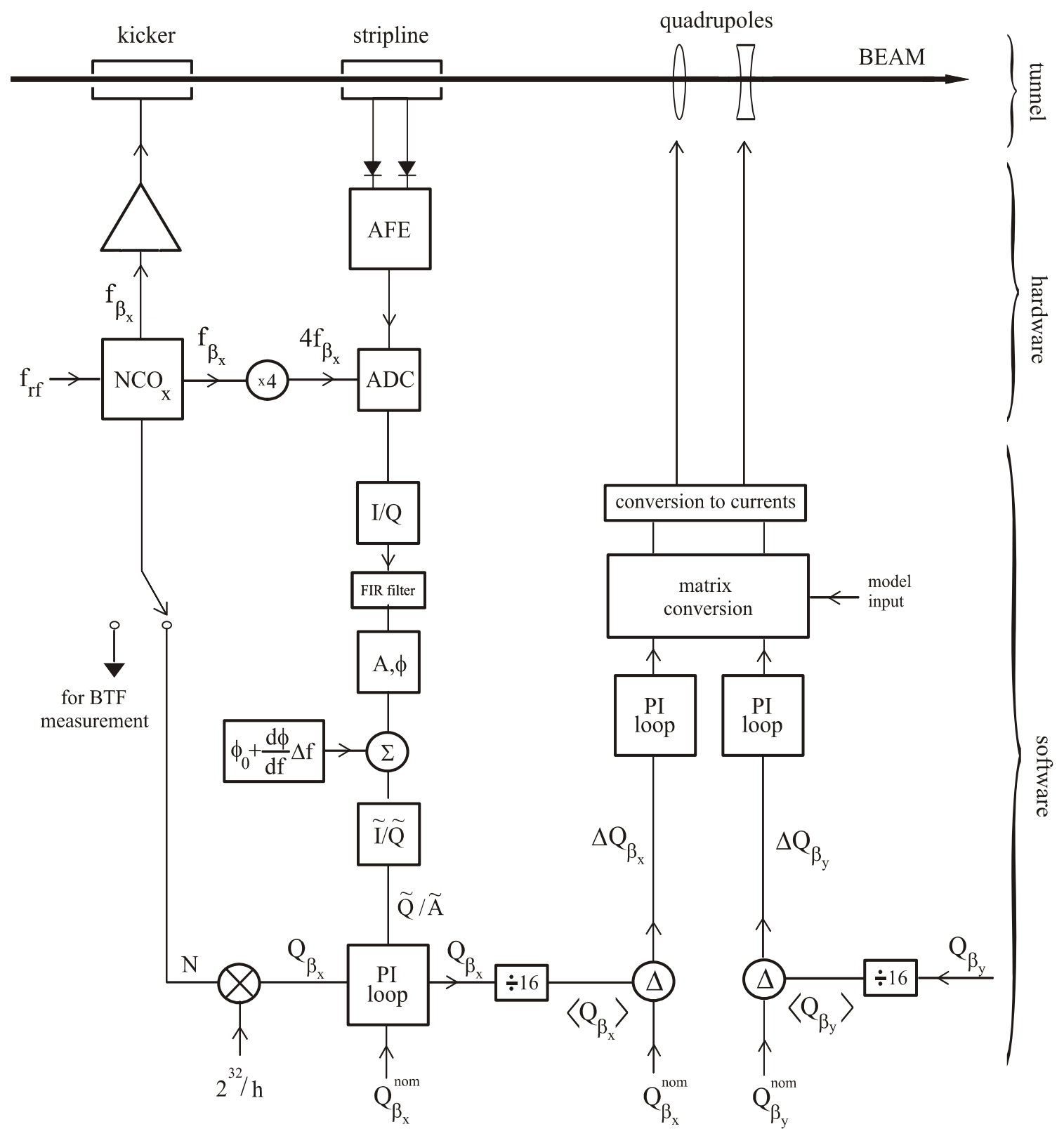

Figure 1: Conceptual sketch of tune measurement and tune feedback in one plane with no coupling with emphasis on signal processing algorithms performed in software.

A conceptual diagram highlighting the essential features for measurement of the betatron coupling is given in Fig. 2. For measurement of the eigen-frequencies with nonzero coupling, for each of the four transverse planes, a duplicate AFE output signal is generated and digitized. In a given accelerator, the digitized signal from one plane is sampled at the betatron frequency of the orthogonal plane. The processing of this signal is then identical to that of the tune measurement. Once the amplitude and phase from the coupled channel $\left(\tilde{\mathrm{A}}_{c}, \phi_{c}\right)$, are determined, these together with those of the orthogonal channel $(\tilde{\mathrm{A}}, \tilde{\phi})$ and those obtained in the other plane $\left(\tilde{\mathrm{B}}_{c}, \tilde{\psi}_{c}\right.$ and $\left.\tilde{\mathrm{B}}_{\mathrm{c}}, \tilde{\psi}_{\mathrm{c}}\right)$, shown entering from the lower right of Fig. 2, are used to compute the amount of rotation of the beam ellipse in the $\mathrm{x}-\mathrm{y}$ plane where, for example, the projections onto the $\mathrm{x}$ and 
$y$-axes represent the measured betatron amplitudes without coupling. The skew quadrupole corrections are derived using a single, model-dependent, parameter to remove this rotation using two nearly orthogonal families of skew quadrupoles [5].

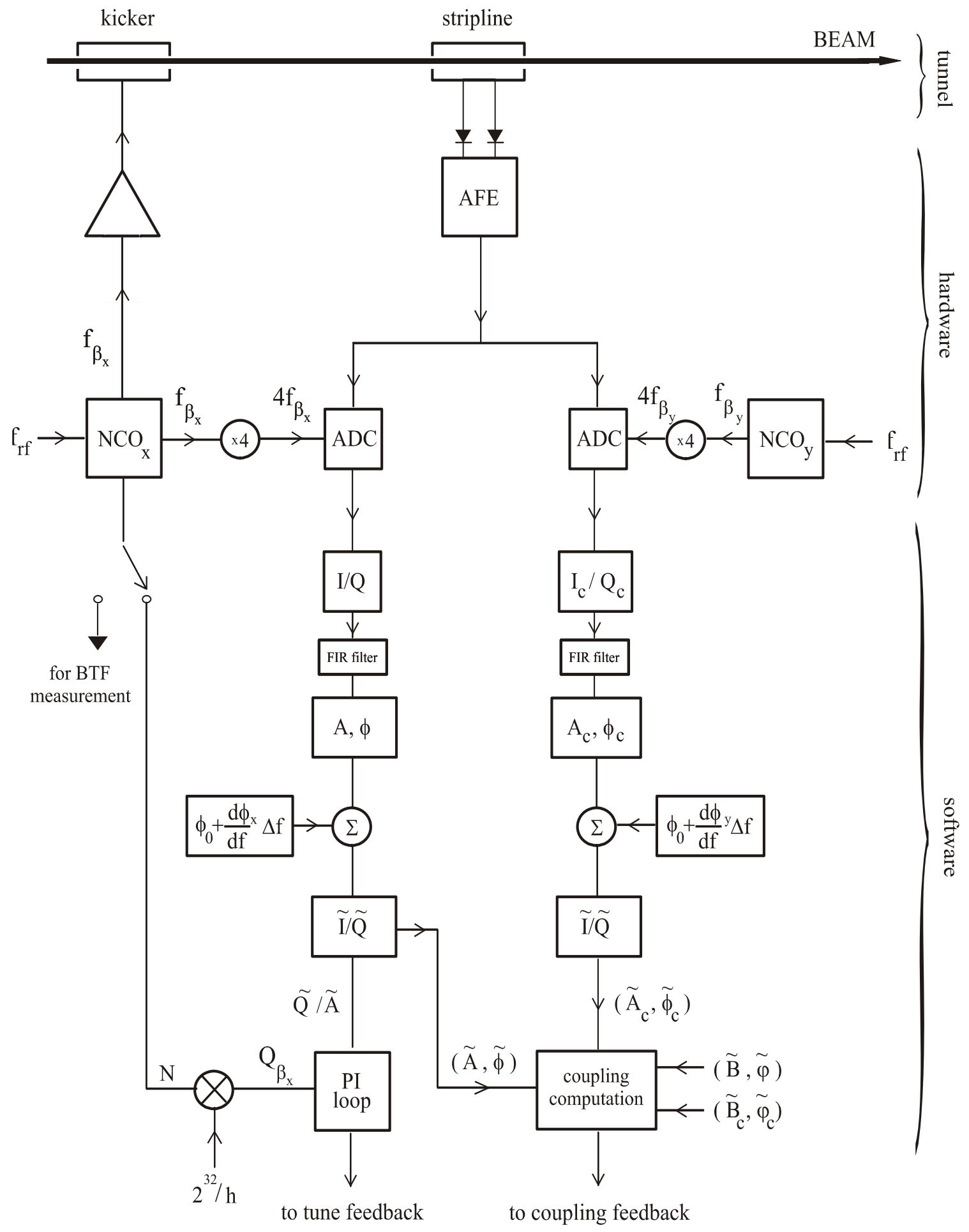

Figure 2: Conceptual diagram of tune measurements with coupling and emphasis on signal processing algorithms performed in software. 


\section{Tune Measurements}

The betatron tunes measured in situ with a variable frequency resonator used to simulate the beam response are shown for the blue and yellow rings in Fig. 3. Also depicted are linear fits through a selected portion (denoted by vertical lines) of the sinusoidal excitation. The resolution determined from the root-meansquare of the residuals between the measured data and linear fit is $4.0 \mathrm{E}-6(0.07 \mathrm{~Hz})$ in the blue ring and $1.8 \mathrm{E}-5(0.33 \mathrm{~Hz})$ in the yellow ring.
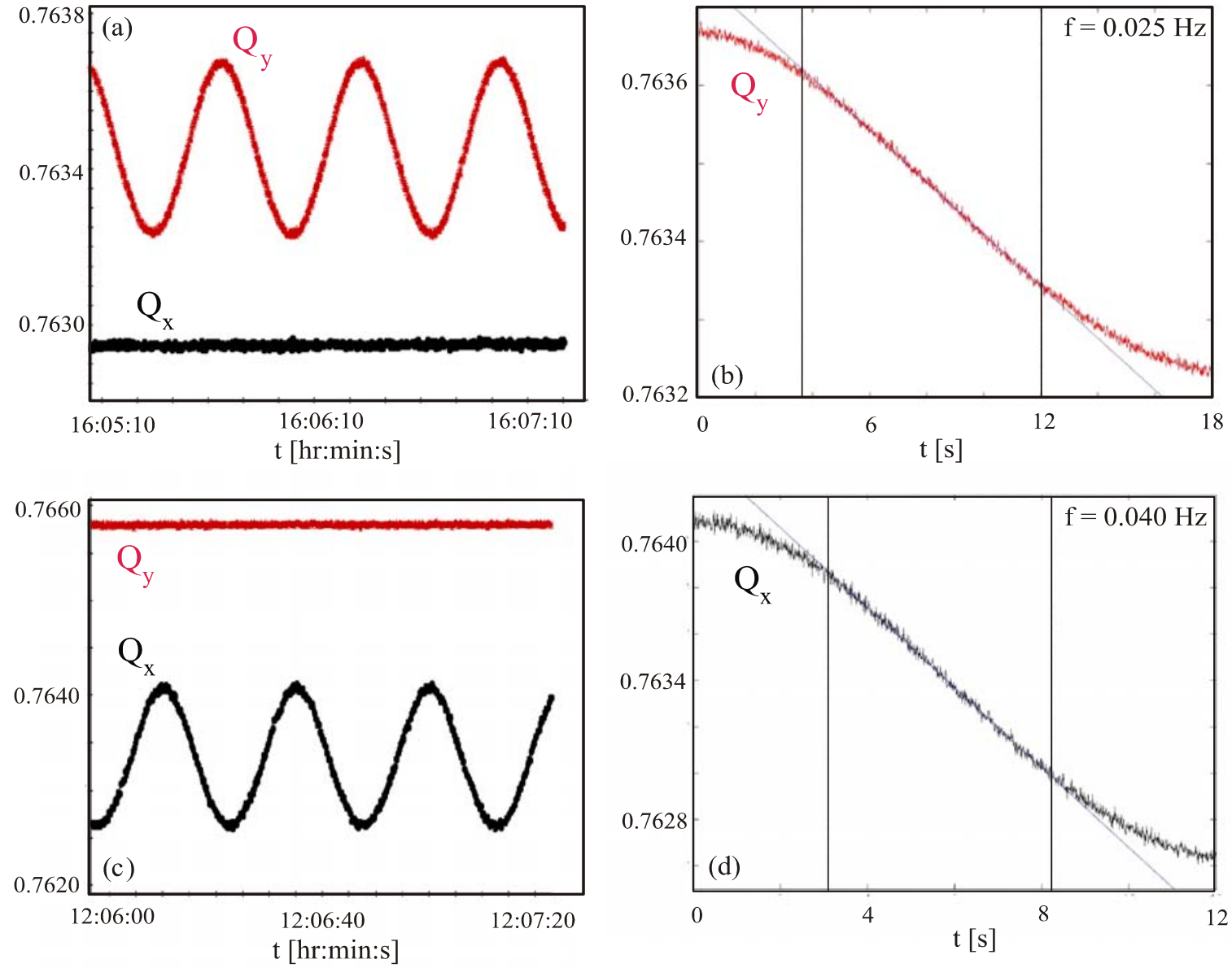

Figure 3: Measured tunes ${ }^{1}$ in the blue ring (a) and the yellow ring (c) with variable frequency resonator input and the same with expanded axis scales (b,d) with linear fit through the region depicted by vertical lines. From the rms deviation between fit and measurement, the resolution of the fractional betatron tunes was measured to be $0.4 \mathrm{E}-5$ in the blue ring and $1.8 \mathrm{E}-5$ in the yellow ring.

Tune measurements with beams at injection energy obtained while modulating the accelerator defocusing quadrupole family are shown from the blue ring in Fig. 4 and from the yellow ring in Fig. 5 with modulation of the focusing quadrupole family. By nature of the layout of the magnets and power supplies, the excitation experienced by the beam in the plane orthogonal to the applied modulation is estimated at $20.3 \%$ while the amplitude ratio between planes was measured to be $\sim 30 \%$ in both rings indicating additional contributions due presumably to a combination of coupling and residual orbit oscillations (feeddown from off-axis beam in the ring sextupole magnets). From the data in the figures, the measured resolution with beams using the deviation from linear fit as before are $8.0 \mathrm{E}-5(1.94 \mathrm{~Hz})$ in the blue ring and $5.6 \mathrm{E}-5(1.32 \mathrm{~Hz})$ in the yellow ring.

${ }^{1}$ Data from 01/16/09 (blue ring), 01/24/09 (yellow ring). 

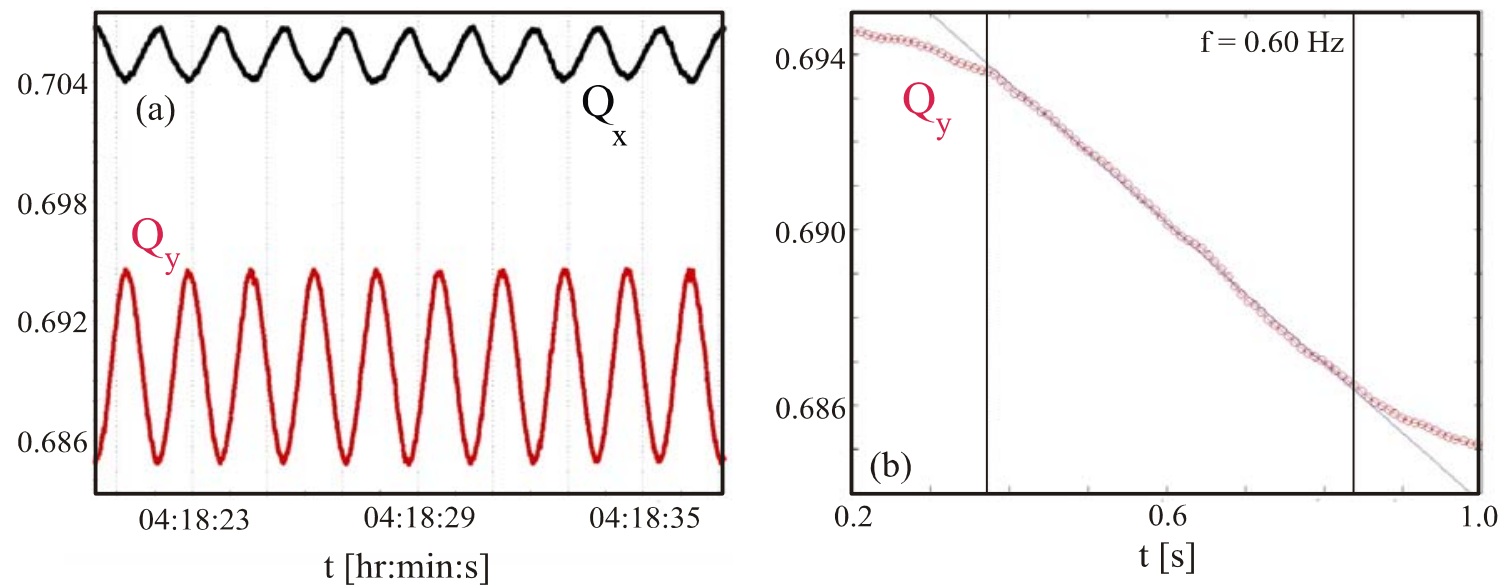

Figure 4: Measured tunes in the blue ring ${ }^{2}$ with (defocusing) quadrupole modulation (a) and the same with expanded axis scales and linear fit through the region depicted by vertical lines in (b). The standard (rms) deviation between fit and measurement is 8.0e-5.
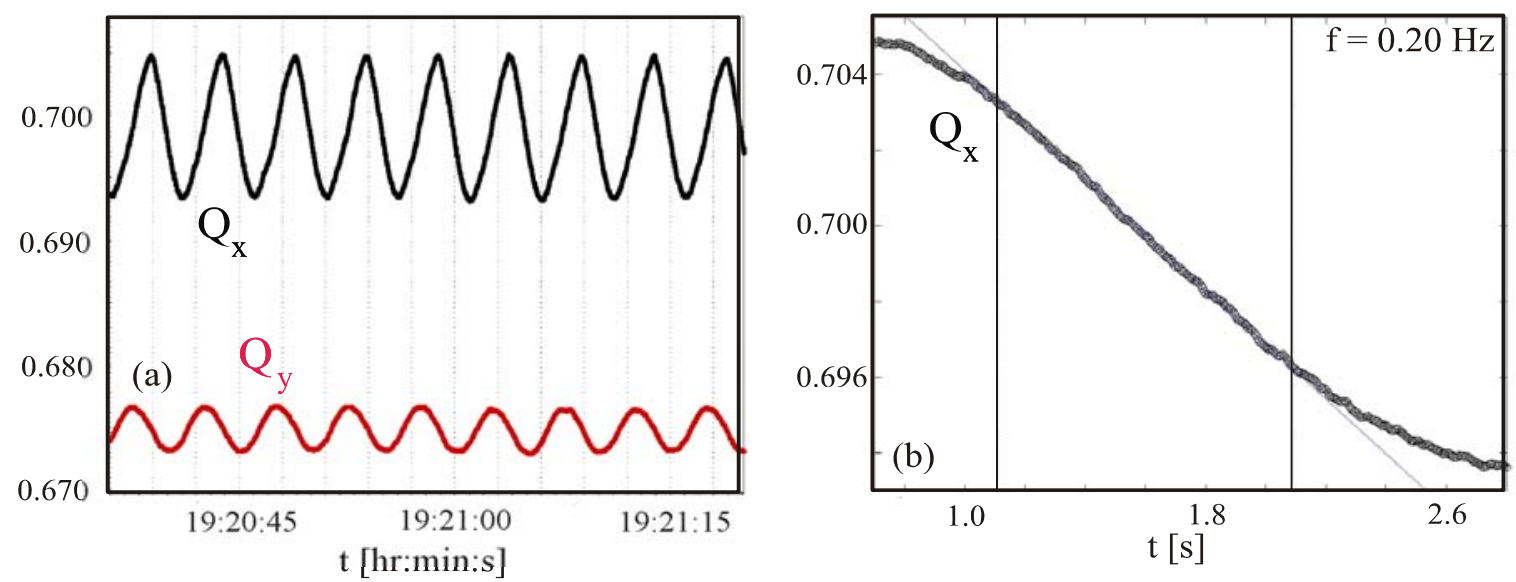

Figure 5: Measured tunes in the yellow ring ${ }^{3}$ with (focusing) quadrupole modulation (a) and the same with expanded axis scales and linear fit through the region depicted by vertical lines in (b). The rms deviation between fit and measurement is 5.6E-5.

During the short time between the time the data of the last two figures were acquired, a significant change to the data processing code, as explained in Section 8, was performed which significantly improved the measurement resolution. The instrumental resolution measured following these changes is illustrated in Fig. 6 where is again shown data measured in situ with a variable frequency resonator. Taking into account now statistical errors on the measurements, the resolution from Fig. 3 is $(4.3 \pm 0.2) \times 10^{-6}$ compared to that of Fig. 6 (present condition) with a resolution of $(1.9 \pm 0.1) \times 10^{-6}$ (or $\left.0.05 \mathrm{~Hz}\right)$.

For some time it has been known that the beam orbits in RHIC are perturbed at frequencies close to $10 \mathrm{~Hz}$ due to vibration of the triplet quadrupoles near the interaction regions [9]. Shortly after first establishing beams at full energies of $250 \mathrm{GeV}$ per beam during Run-9, significant tune modulations were observed for both beams in both planes. The tune modulations are believed to result from quadrupole fields sampled by off-axis trajectories (made so by the vibrating quadrupoles) in higher-order multipole fields particularly in the ring sextupoles. Shown in Fig. 7 are the measured tunes in the blue ring obtained under store conditions in both the transverse planes. The data are also shown with expanded view from which the

${ }^{2}$ Data from 02/13/09, fill 10049.

${ }^{3}$ Data from 02/14/09, fill 10050. 
component at $\sim 10 \mathrm{~Hz}$ is clearly visible with surprisingly large amplitudes of about 1.0E-3 peak-to-peak in both transverse planes. The plots on the left show additionally a modulation of the $10 \mathrm{~Hz}$ envelop arising presumably due to beating of two nearby excitation frequencies.
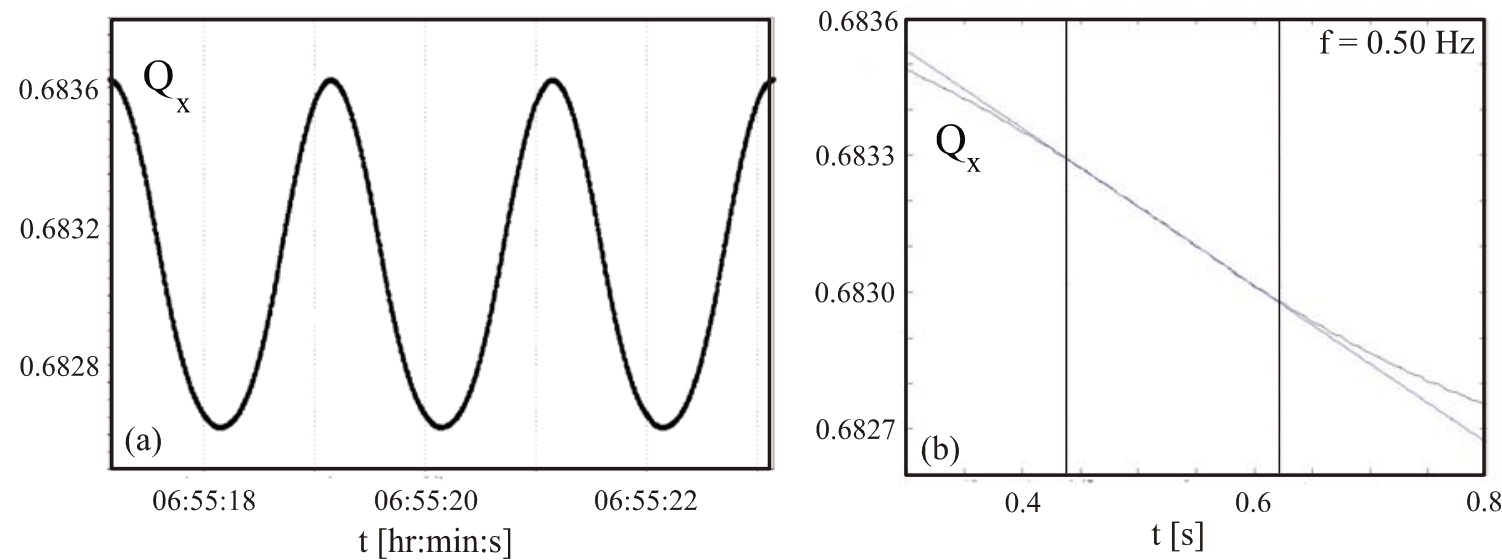

Figure 6: Measured tune in the blue ring with variable frequency resonator input (a) and the same with expanded axis scales (b) acquired at the end of Run-9 following the many hardware and software improvements described in sections 7 and 8 evidencing an instrumental resolution of $(1.9 \pm 0.1) \times 10^{-6}$.
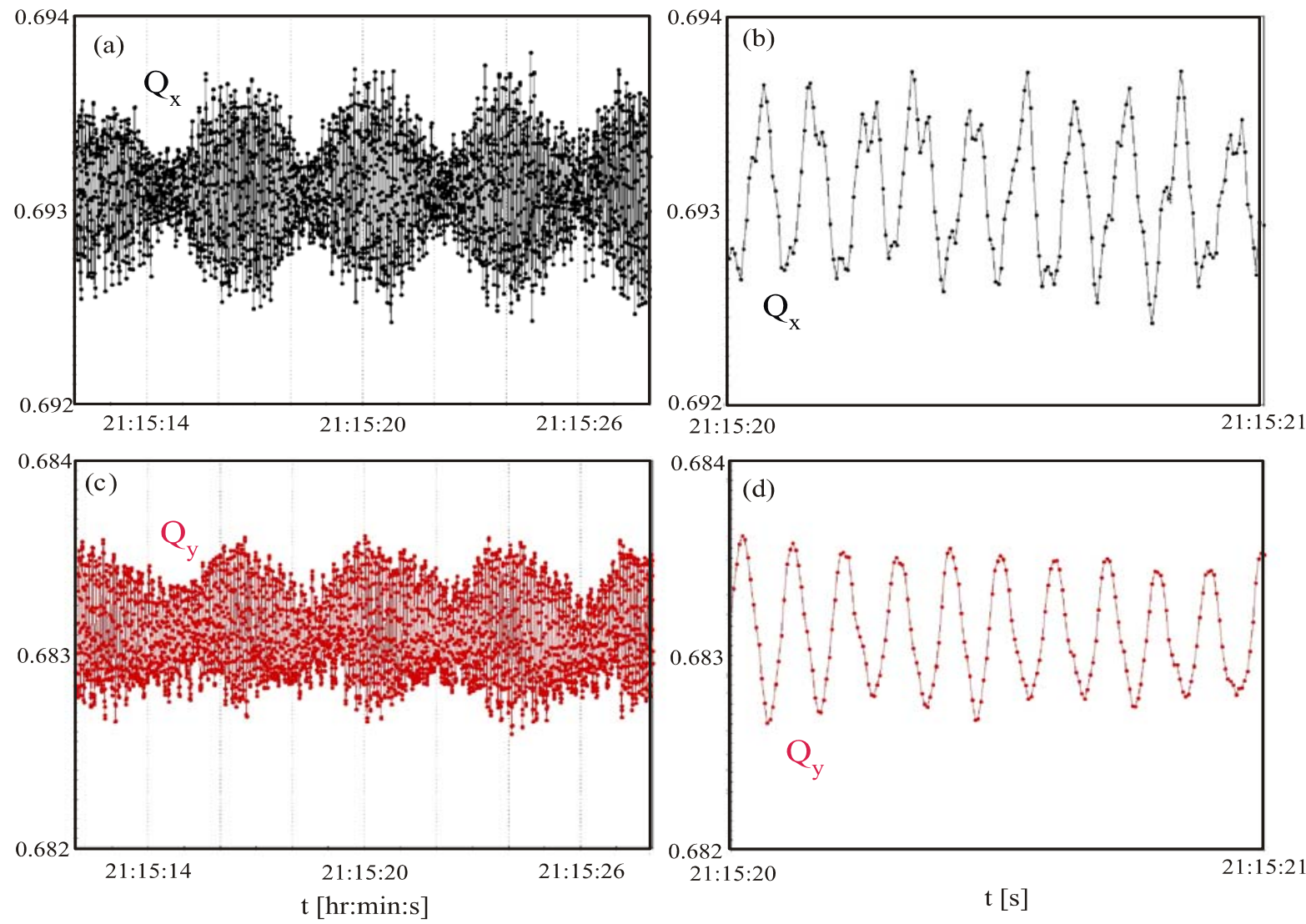

Fig. 7: Measured ${ }^{4}$ under store conditions in the blue ring at $250 \mathrm{GeV}$, first observation of strong $\sim 10 \mathrm{~Hz}$ modulation of the betatron tunes. Plotted are the horizontal (a) and vertical (c) tunes and the same with expanded time scale on the right.

${ }^{4}$ Data from 02/20/09, fill 10166. 
In Fig. 8 are shown the betatron frequencies measured during the energy ramp as the design tunes crossed. In a given ring, the oscillations are observed to be out of phase between the two transverse planes which suggests strongly that the observed tune modulations are not instrumental. The data also show that in a given plane (horizontal or vertical), the oscillations are in phase suggesting a dominant driving term common to both accelerators. Lastly we note that the distance of closest approach of the times, a parameter characterizing the degree of global coupling, may be directly determined from these data.
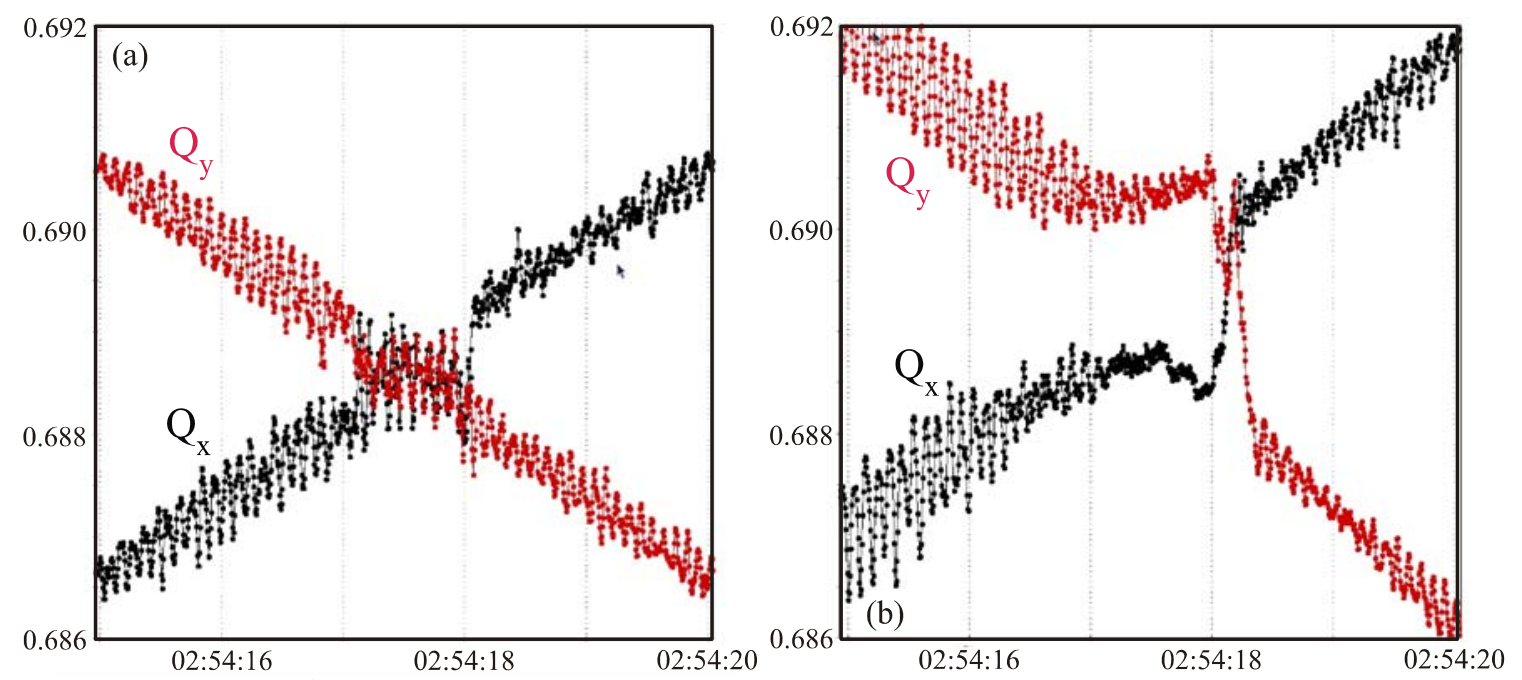

Figure 8: Measured ${ }^{5}$ during the energy ramp, the betatron tunes in the blue (a) and yellow (b) rings evidencing three notable features as described in the text.

Conclusive evidence for tune modulation being caused by the vibrating triplet magnets is presented in Fig. 9. The Fast Fourier Transform (FFT) of the horizontal beam position (BPM) sampled at a rate of $720 \mathrm{~Hz}$ is shown in Fig. 9a while the FFT of the measured horizontal tune, sampled at approximately $185 \mathrm{~Hz}$, is shown in Fig. 9b. The data are presented with expanded frequency axes in the lower portion of Fig. 9. Of these, the left two subplots span $5-10 \mathrm{~Hz}$ centered about the dominant frequencies as measured by mechanical accelerometers and geophones detecting local mechanical displacements [10] while the two plots on the right span 8-12 $\mathrm{Hz}$ centered about those frequencies having dominant influence on the beam orbit and optics. The discrete frequencies detected by the BPMs and tune monitor are identical which points unambiguously to a common source. While not shown, we remark that measurements of the vertical beam position and vertical betatron tunes measured simultaneously to the data shown revealed excitations at the same frequencies as those measured in the horizontal plane.

The influence of the tune modulations, which were curiously absent at the start of Run-9, persisted throughout the remainder of the running period. In many cases, the resolution of the tune measurement was entirely dominated by the modulations as shown in Fig. 10. These data (blue ring, top; yellow ring, bottom) were acquired at injection during rf frequency modulation at a $2 \mathrm{~Hz}$ rate moments before the energy ramp for measurements of the chromaticity. In this case, and many others, the peak-to-peak amplitude of the tune modulation is seen to exceed by at least a factor of 10 the measurement resolution.

For applications requiring precision tune measurements, the $\sim 10 \mathrm{~Hz}$ modulations may be filtered out. Presented in Fig. 11 are measurements obtained during correction of nonlinear fields in the interaction region from the yellow ring. Plotted is the absolute value of the measured tune deviation from nominal as a function of the amplitude of a horizontal bump which was varied in a sinusoidal fashion. The tune measurements were made with 1 second averaging to filter the variations due to the $\sim 10 \mathrm{~Hz}$ modulations. In earlier studies ${ }^{6}$ a factor of (2-3) reduction in tune modulation amplitude was observed following the application of nonlinear corrections using precision tune measurements.

\footnotetext{
${ }^{5}$ Data from 03/18/09, fill 10384.

${ }^{6}$ Data from 03/31/09, fill 10466.
} 

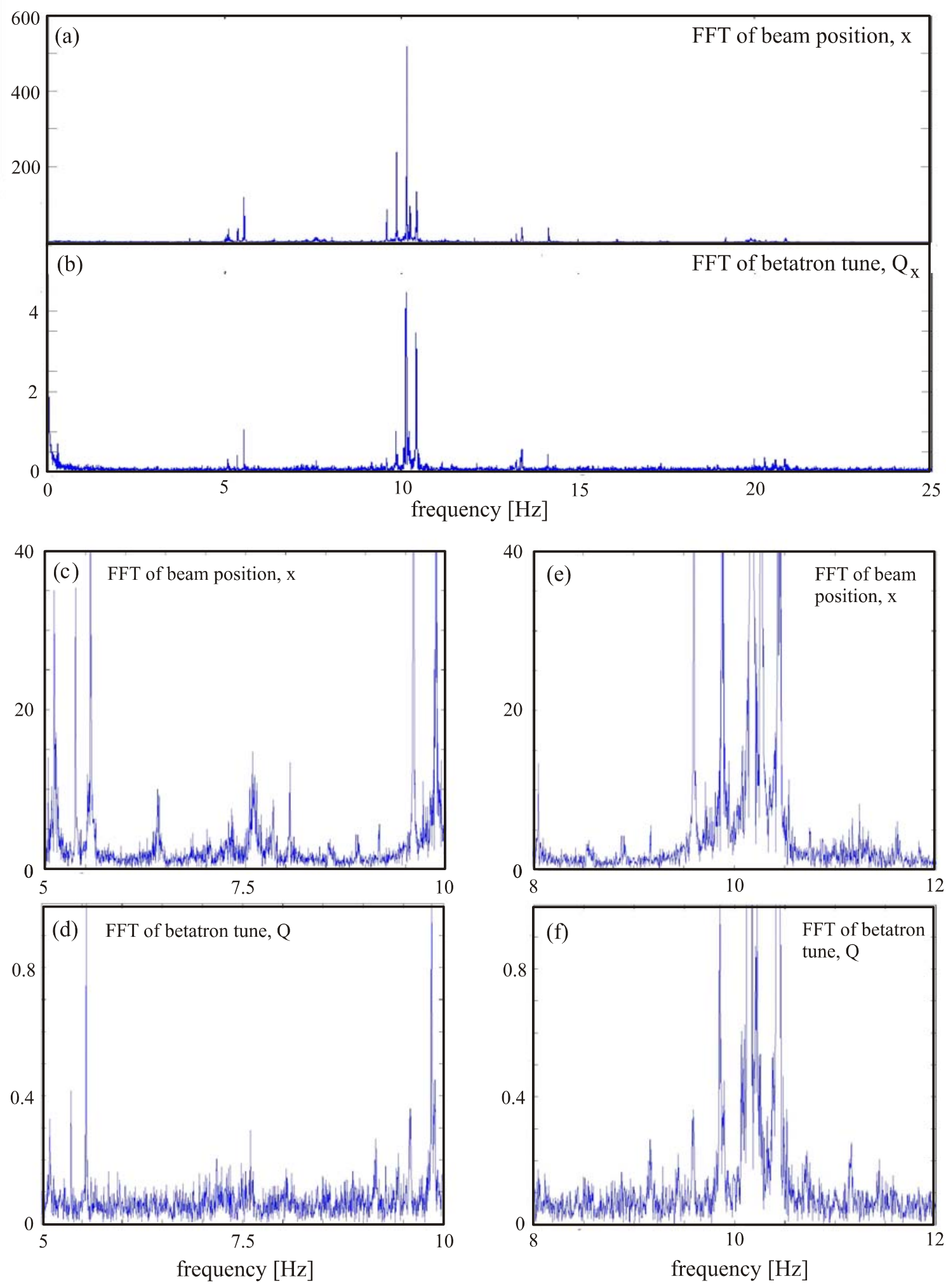

Figure 9: Fourier Transforms of the horizontal beam position (a,c,e) and horizontal betatron tune (b,d,f) as measured $^{7}$ in the blue ring with horizontal scales of $0-25 \mathrm{~Hz}$ (top) and expanded views with span 5-10 Hz, encompassing known mechanical resonant frequencies, and 8-12 Hz below.

${ }^{7}$ Data from 02/20/09, fill 10166. 

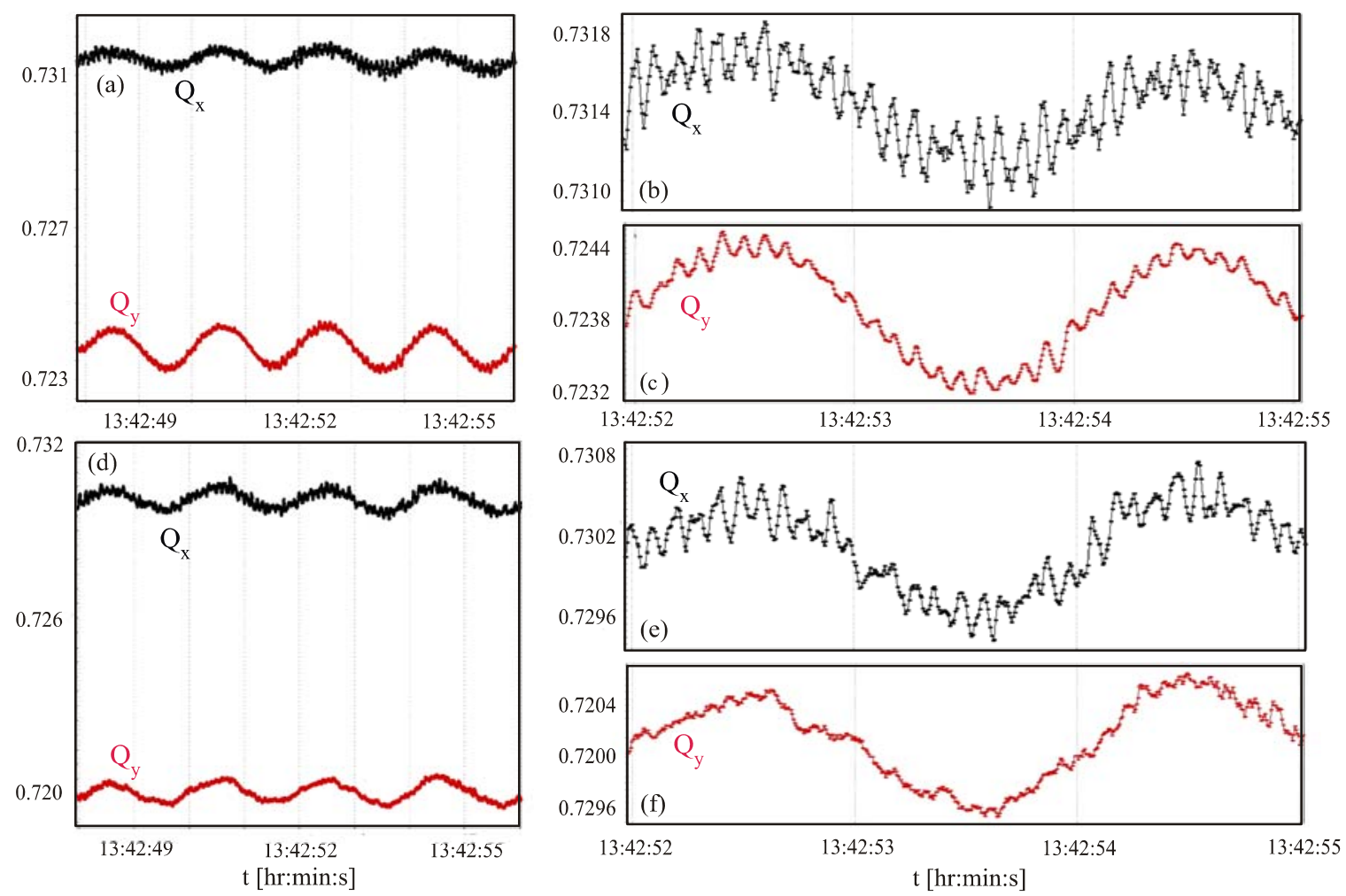

Figure 10: Measured $^{8}$ at injection energy, the betatron tunes in the blue ring (a) and yellow ring (b) during rf frequency modulation at a $0.5 \mathrm{~Hz}$ rate in preparation for chromaticity measurements during the energy ramp. Expanded views of the individual tunes evidencing strong additional modulations at $\sim 10 \mathrm{~Hz}$ are shown on the right: blue ring horizontal (b), blue ring vertical (c), yellow ring horizontal (e), yellow ring vertical (f).

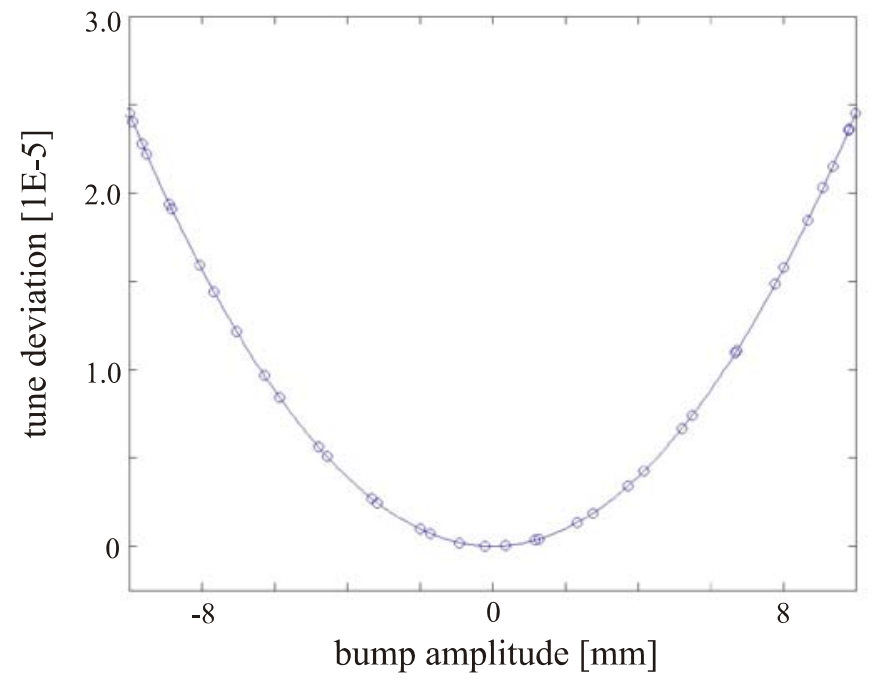

Figure 11: Tune deviation with $\sim 10 \mathrm{~Hz}$ filtering measured ${ }^{9}$ versus horizontal bump amplitude applied through the interaction region during measurement characterizing local nonlinearities. Note the $10^{-5}$ vertical scaling factor.

${ }^{8}$ Data from 04/20/09, fill 10631.

${ }^{9}$ Data from 07/06/09, fill 11066. 


\section{Coupling Measurements}

Prior to initial ramp development with tune and coupling feedback, the global betatron coupling of each beam is minimized at injection energy using tune measurements acquired while modulating the coupling angle [11]. Example data acquired in the Yellow Ring are shown in Fig. 12 (top two plots, Run-9; bottom two plots, Run-8) where the magnitude of the required correction strengths, $\kappa_{1}$ and $\kappa_{2}$, inferred directly from measurements of the betatron tunes are given as a function of time (see [11]). Perfect decoupling is achieved when modulation of one family of skew quadrupoles has no effect on the data acquired in the orthogonal plane. Note the factor of $>2.5$ decrease in the vertical scale between data sets. Taking as a measure of coupling control the rms of the signal strength in the orthogonal plane in (b) and (d), the precision on the control of the decoupling was reduced from +/- 0.0414 in Run-8 to +/- 0.0035 in Run-9.
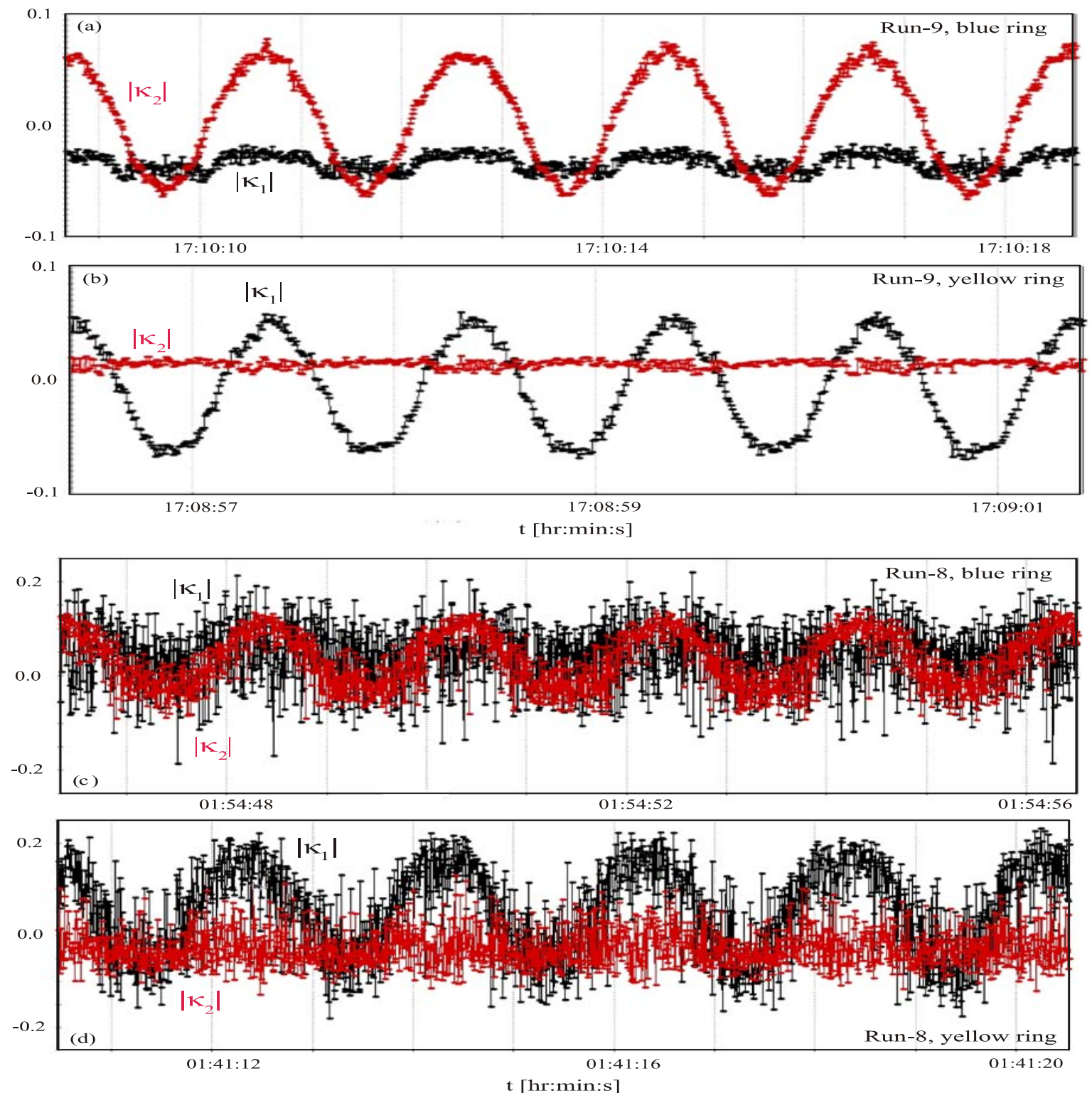

Figure 12: Run- $9^{10}(\mathrm{a}, \mathrm{b})$ and Run- $8^{11}(\mathrm{c}, \mathrm{d})$ coupling coefficients as measured at injection with applied modulation of coupling angles. Note the change in vertical scale.

${ }^{10}$ Data from 06/10/09, fill 10892.

${ }^{11}$ Data from 01/31/08, fill 9670. 


\section{Performance of Tune and Coupling Feedback}

Tune and coupling feedback were successfully executed from the onset of ramp development for Run-9 beginning with record minimum times of one shift per ring as consecutively commissioned, based on accelerator availability, to accelerate proton beams to their full energies of $250 \mathrm{GeV}$. Summarized in Fig. 13 are accelerator development periods with feedback used for commissioning of different beam energies and/or optics, optimizing betatron tunes during the energy ramp for high polarization transmission efficiency (particularly during the $250 \mathrm{GeV}$ run) or for intensity transmission efficiency (during the 100 GeV run). Table 1 summarizes the development periods which are presented in more detail in Appendix A.

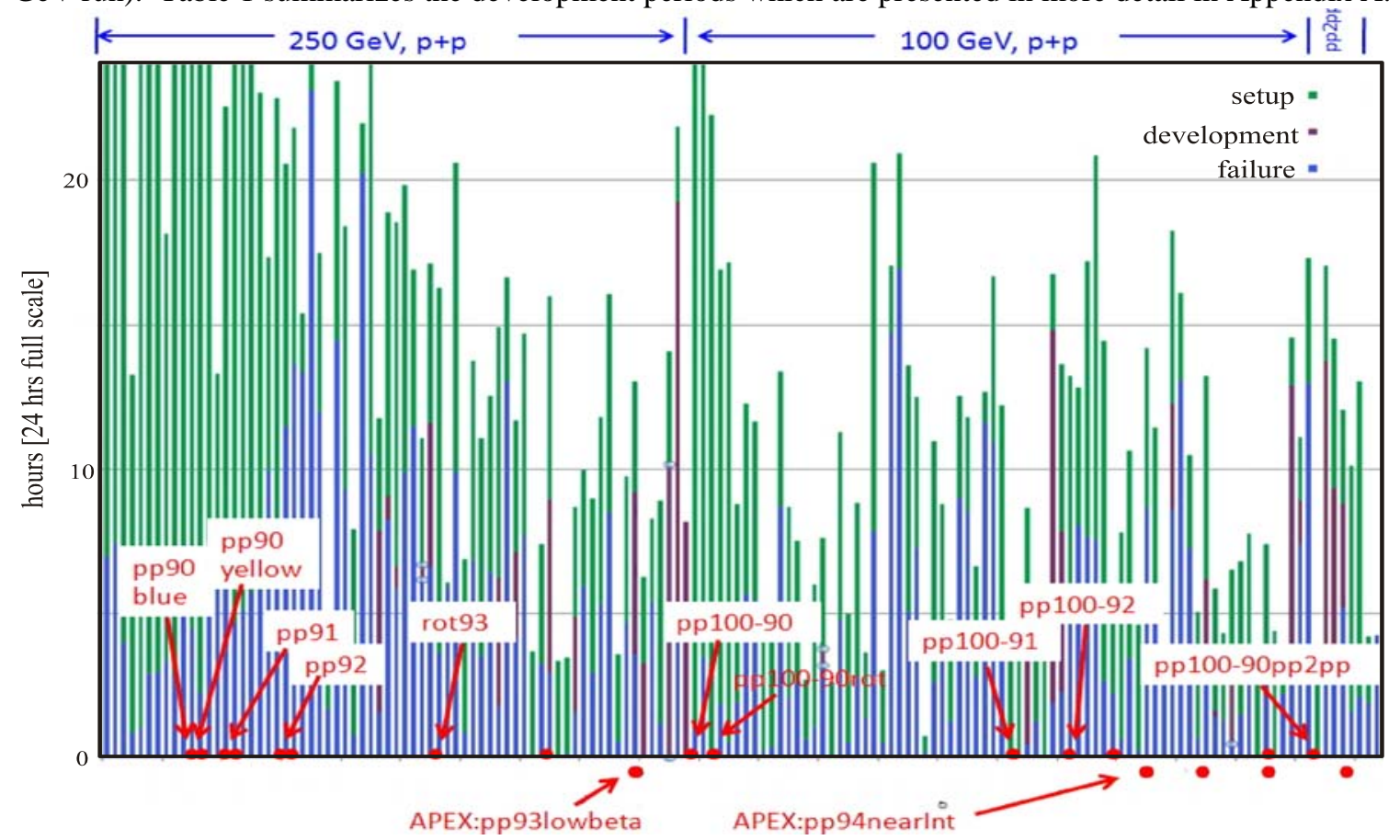

Figure 13: Overview of ramp development periods with tune and coupling feedback during Run-9. Highlighted are the times of implementation of tune and coupling feedback (red circles), the associated beam optics being commissioned (e.g. pp90) and the times of dedicated accelerator physics experiments denoted by "APEX". Each vertical bar represents one day and the time (horizontal axis) spans 02/06/09 through 07/06/09. The statistics given in the histogram are courtesy P. Ingrassia.

\begin{tabular}{|l|c|c|c|}
\hline mode of operation & dates & $\begin{array}{c}\text { number of } \\
\text { sessions }\end{array}$ & $\begin{array}{c}\text { time to first replay } \\
\text { (hours) }\end{array}$ \\
\hline $250 \mathrm{GeV}, \mathrm{p}^{+} \mathrm{p}$ & $02 / 06 / 09-04 / 14 / 09$ & 10 & 8 per accelerator \\
$100 \mathrm{GeV}, \mathrm{p}+\mathrm{p}$ & $04 / 15 / 09-06 / 29 / 09$ & 6 & 4 \\
$100 \mathrm{GeV}, \mathrm{p}+\mathrm{p} . \mathrm{pp} 2 \mathrm{pp}$ & $06 / 29 / 09-07 / 04 / 09$ & 1 & 4 \\
$250 \mathrm{GeV}, \mathrm{p}+\mathrm{p}, \mathrm{APEX}$ & $06 / 10,16-17,24 / 09,07 / 04 / 09$ & 4 & 8 \\
\hline
\end{tabular}

Table 1: Summary of ramp development periods used per mode of operation and the time required to first establish beams to full energy using the quadrupole and skew quadrupole currents as obtained from tune and coupling feedback.

Precision measurement and control of the tunes and coupling is demonstrated clearly by comparison of the measured betatron tunes and the coupling strengths in Fig. 14 (Run-9) with tune and coupling feedback and Fig. 15 (Run-8), a run for which tune and coupling feedback with "tune swing” was only partially achieved. 

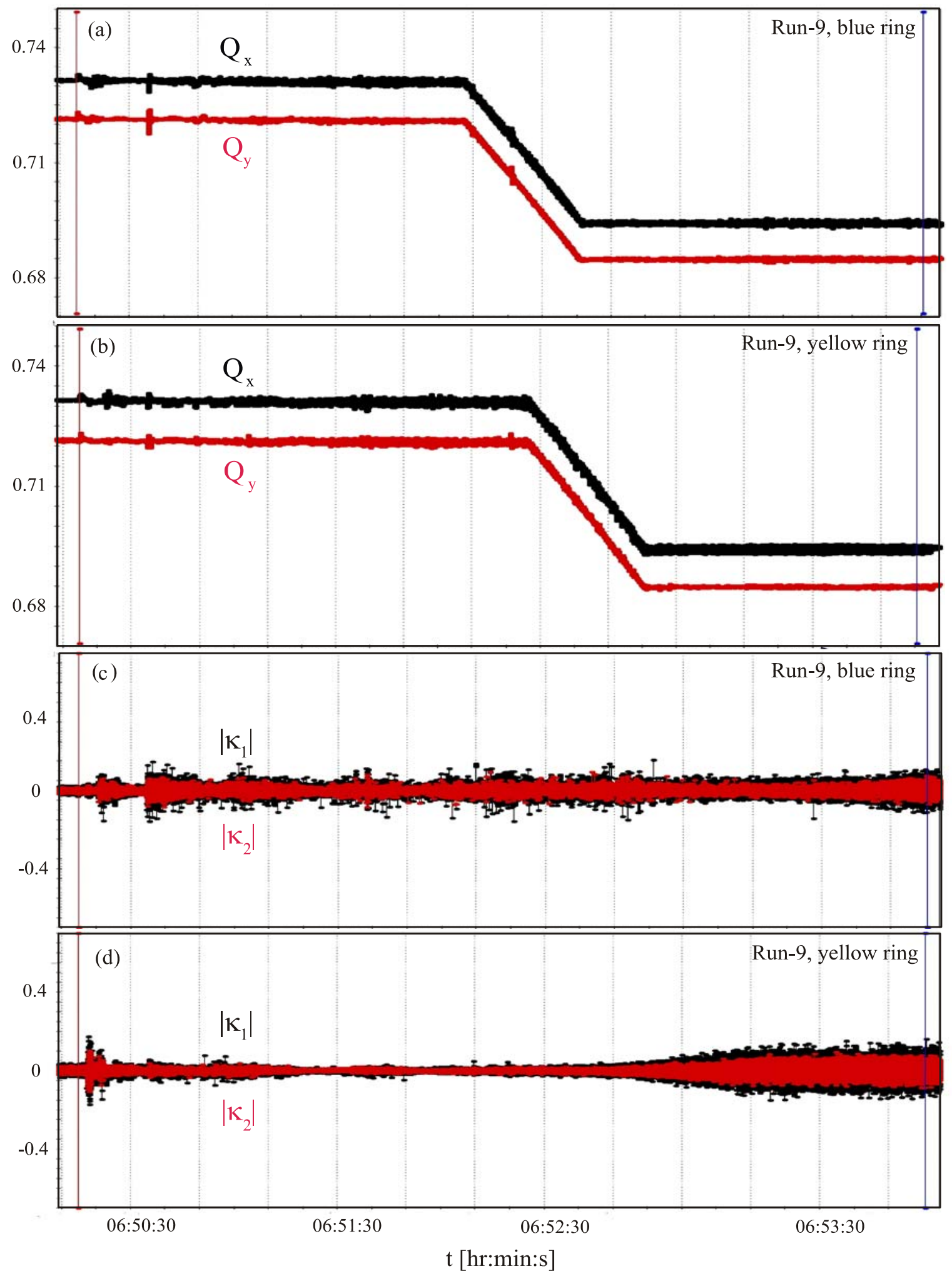

Figure 14: Run-9 measured ${ }^{12}$ betatron tunes with "tune swing" with $100 \mathrm{GeV}$ proton beams in the blue (a) and yellow (b) rings and measured coupling strengths in the blue (c) and yellow rings (d).

${ }^{12}$ Data from 04/16/09, fill 10568. 

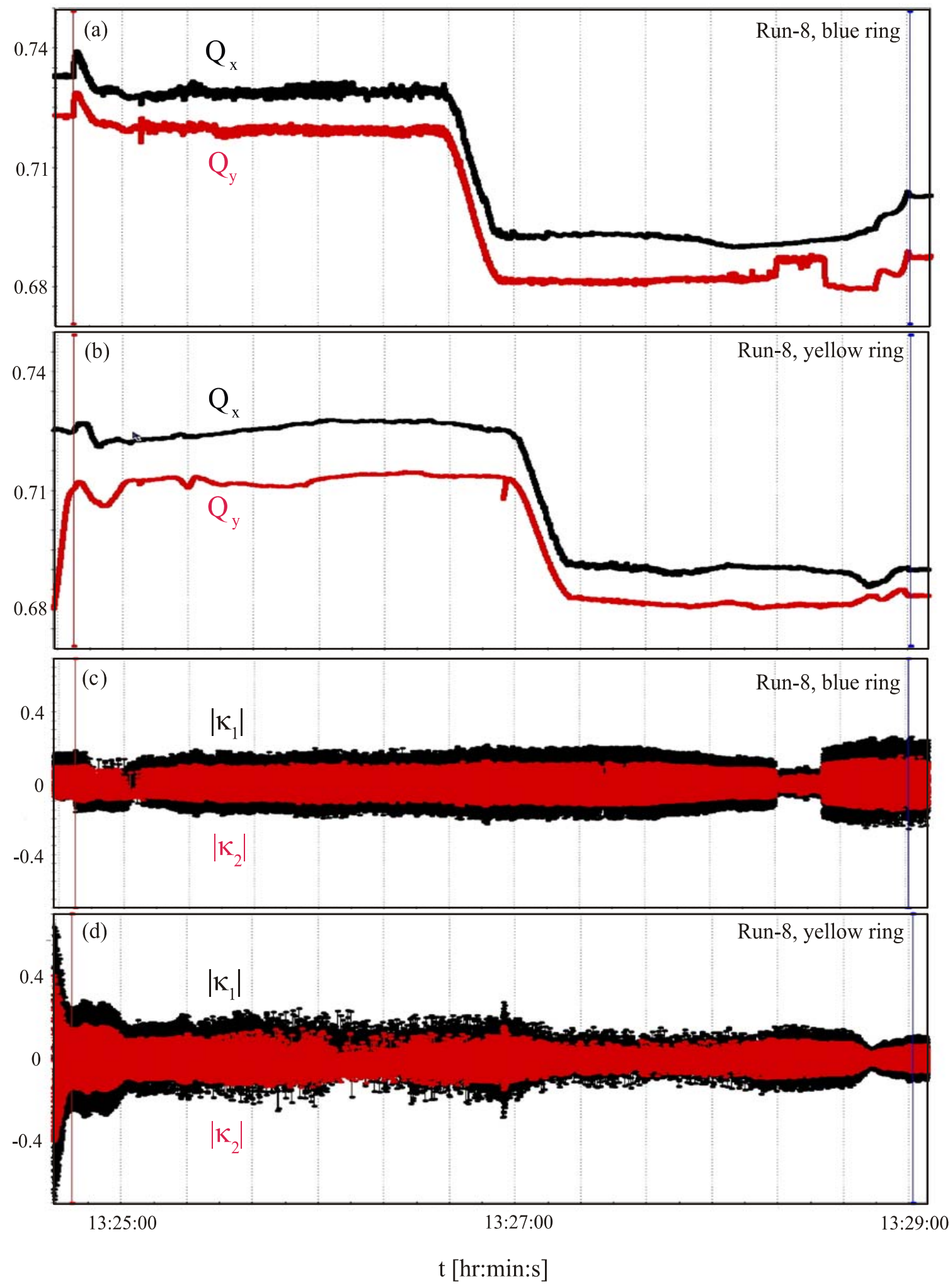

Figure 15: Run- 8 measured ${ }^{13}$ betatron tunes with "tune swing" with $100 \mathrm{GeV}$ proton beams in the blue (a) and yellow (b) rings and measured coupling strengths in the blue (c) and yellow rings (d).

${ }^{13}$ Data from 02/21/08, fill 9922. 
We remark that, starting from the beginning of Run-9, the execution of tune and coupling feedback was fully automated requiring no human interventions as was needed previously (controlling kicker amplitudes, the phasing of the I/Q demodulator inputs, tune scan ranges for the phase locked loop, closed-loop gain parameters, etc.) during execution. The possibility of human error during ramp development with tune/coupling feedback was therefore substantially reduced.

During Run-9 detailed analyses were made to determine the cause of any ramp with tune and coupling feedback which did not result in a complete record of quadrupole and skew quadrupole currents with tunes controlled to the desired set values and coupling corrected suitable for feed-forward on a subsequent ramp. In histogram format are shown in Fig. 16 the causes for such "failed ramps". Of note are that it was possible to diagnose each and every failed ramp and that the leading cause for failure was due to uncontrolled orbit excursions. These were easily diagnosed using logged parameters which showed increasing rms orbit amplitude followed by beam loss which in turn was followed by loss of tune regulation when the beam current became less than the measurement sensitivity of about 1.5E11 particles corresponding to approximately $1 / 8^{\text {th }}$ of the initial beam intensity used in ramp development. This is a considerable improvement over past years where "loss of lock" of the phase lock loop was considered and might possibly have been the dominant source for failed ramps. A post run performance analysis and further details are given in Appendix B.

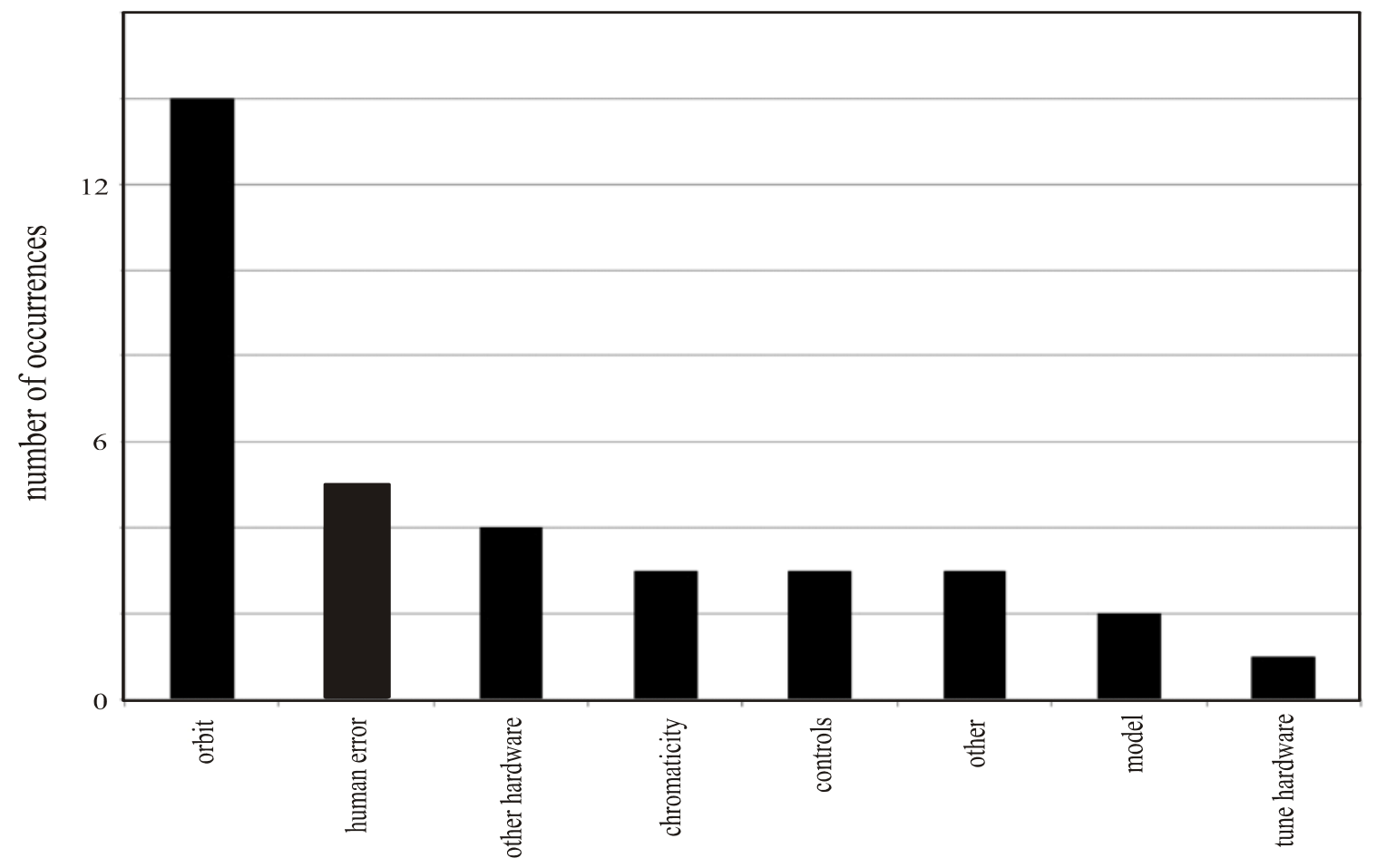

Figure 16: Histogram of causes for "failed ramps" (those not achieving a complete record of quadrupole and skew quadrupole currents with tunes controlled to desired set values and coupling corrected for subsequent feed-forward) during Run-9. A total of 88 ramps were performed with tune and coupling feedback of which 35 were considered to have failed.

A smoothing algorithm may be applied to filter out fast changes in the requested currents as determined during the previous ramp with tune and coupling feedback. During one commissioning period the strengths recorded during tune and coupling feedback contained an instance of misinformation delivered when the phase locked loop locked onto a high harmonic of $60 \mathrm{~Hz}$. These data were post-processed by interpolation across the short region of interest. 


\section{Beam Transfer Function Measurements}

Beam transfer function (BTF) measurements at RHIC are presently made using the same experimental hardware as for the tune measurements with the exception that excitation frequency is independently controlled. Under test conditions using a swept frequency resonator to simulate the beam, the response through the detection system was documented as shown in Fig. 17 for all four transverse planes. Typical system linearity is shown in Fig. 18 where the measured peak amplitude, $A_{x}$ and $A_{y}$, in the horizontal and vertical planes respectively, is plotted as a function of applied kicker drive amplitude.
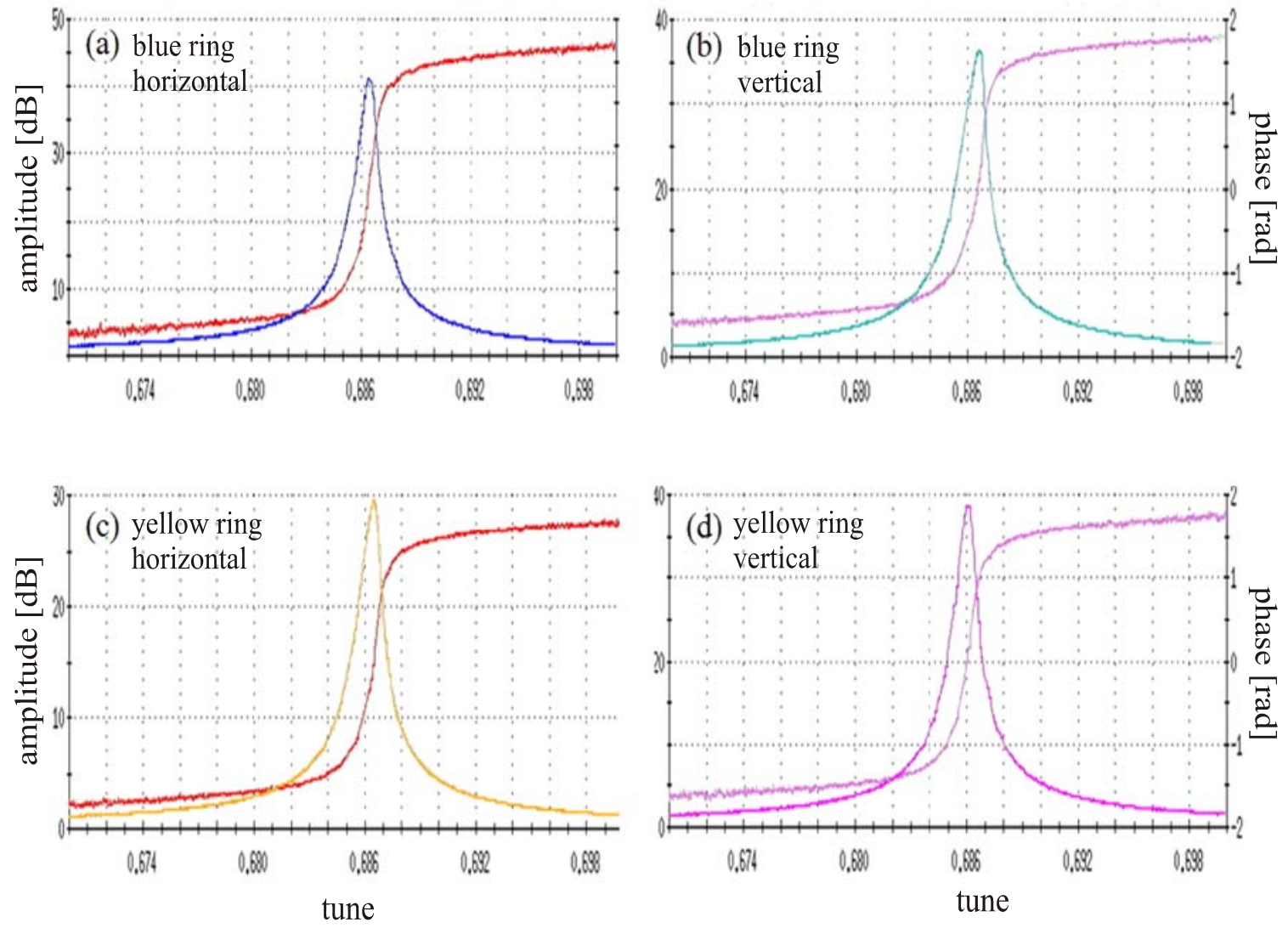

Figure 17: Beam transfer functions (amplitude and phase) measured in situ versus excitation frequency converted to tune units obtained with a variable frequency resonator in the horizontal $(\mathrm{a}, \mathrm{c})$ and vertical (b,d) planes of the blue and yellow rings respectively.
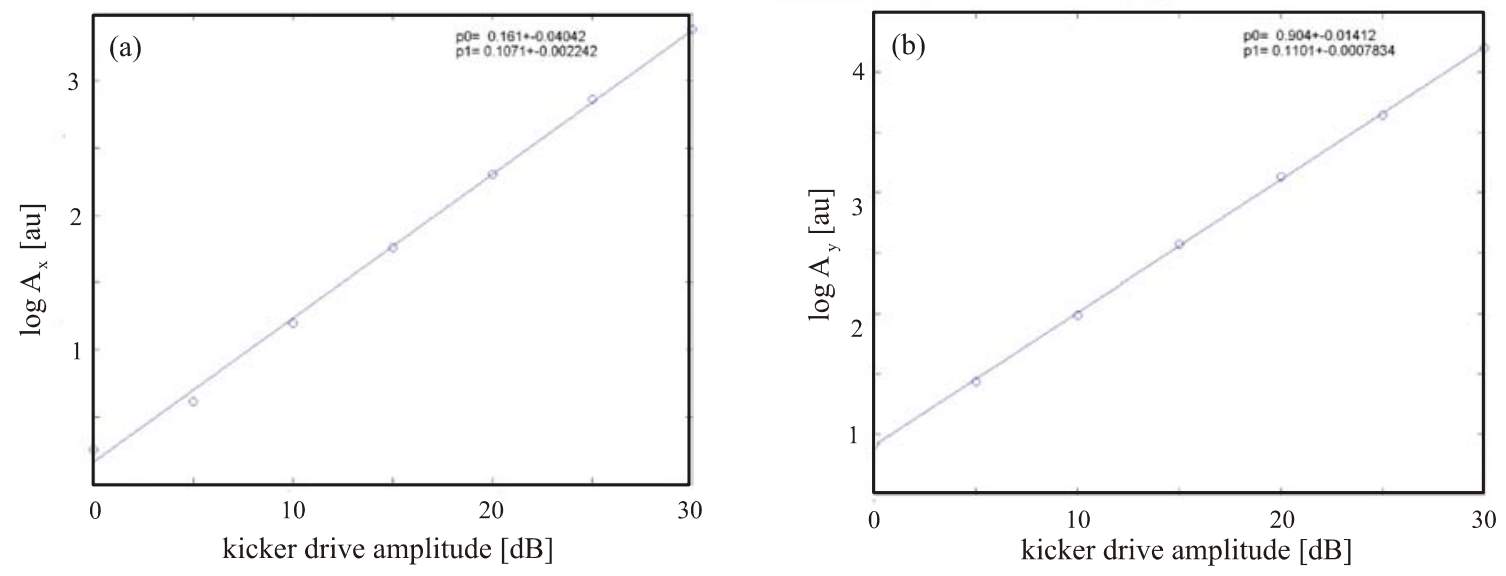

Figure 18: Linearity of the beam transfer function measurements shown in the horizontal (a) and vertical (b) planes of the yellow ring obtained in situ with resonator input. 
The beam response measured with non-colliding beams is shown in Fig. 19 which compares the beam transfer functions measured during Run-6 (left column), reproduced from the only report to date with documented measurements [12] obtained with $100 \mathrm{GeV}$ protons and Run-9 (right column) with protons at $250 \mathrm{GeV}$. In the recent data the total phase shift is $\pm \frac{\pi}{2}$ and the phase is constant where the amplitude response is small as expected.
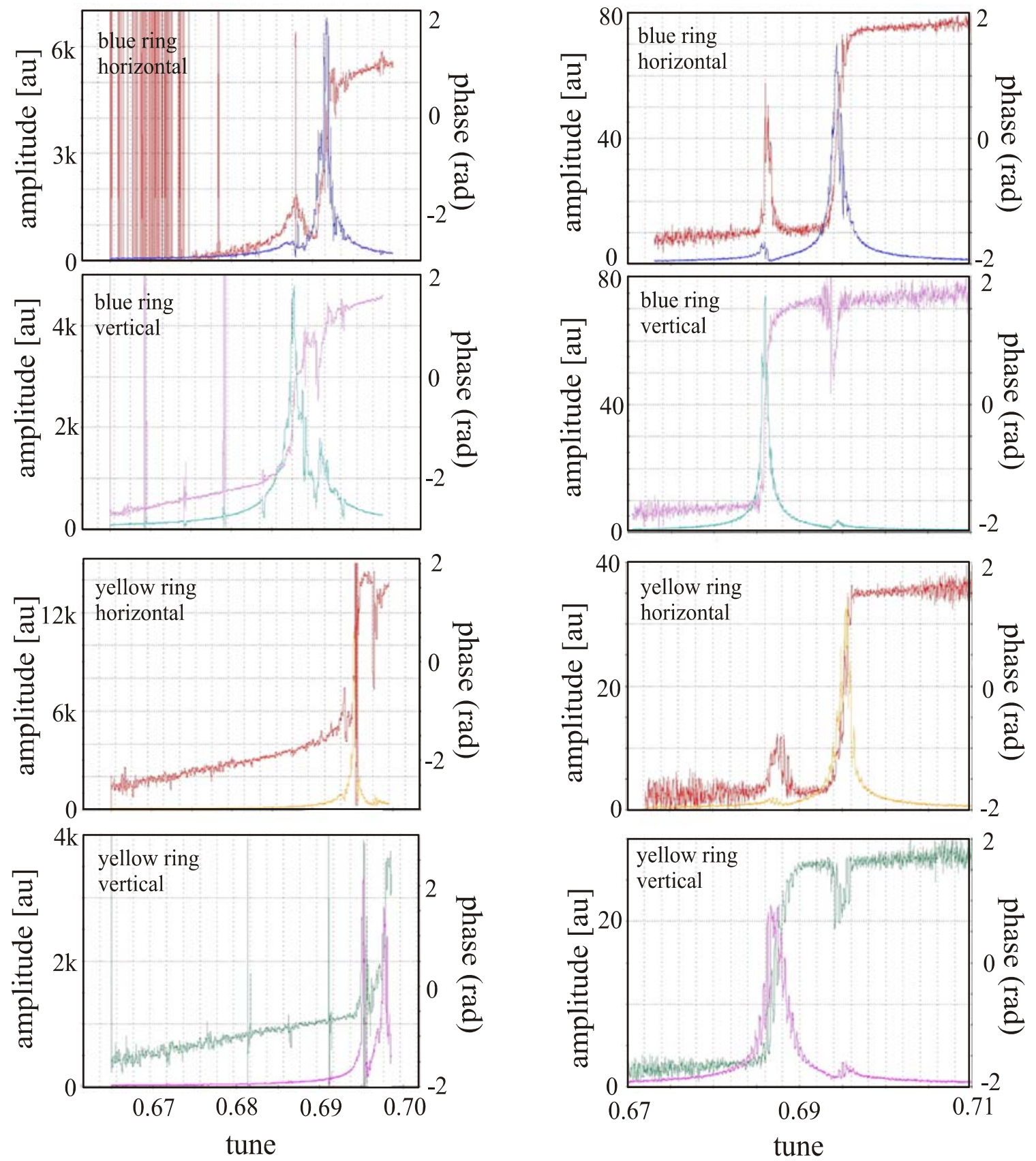

Figure 19: Beam transfer function measurements with non-colliding beams from Run- $6^{14}$ (left) and Run- $9^{15}$ (right) showing amplitude (left axis) and phase responses (right axis). Note the expected $\pm \frac{\pi}{2}$ phase shift at resonance and the flat phase response away from resonance in the Run-9 data.

${ }^{14}$ Data from 05/26/06, fill 7915.

${ }^{15}$ Data from 02/26/09, fill 10240. 
Shown in Fig. 20 are two consecutive beam transfer function measurements obtained with $250 \mathrm{GeV}$ proton beams before and immediately after the beams were brought into collision. Before collisions the betatron tunes were similar in the two rings: in the blue ring: $\mathrm{Q}_{\mathrm{x}}=0.6942, \mathrm{Q}_{\mathrm{y}}=0.6860$; in the yellow ring: $\mathrm{Q}_{\mathrm{x}}=0.6965$, $\mathrm{Q}_{\mathrm{y}}=0.6870$ or $\left|\Delta \mathrm{Q}_{\mathrm{x}}\right|=0.0023$ and $\left|\Delta \mathrm{Q}_{\mathrm{y}}\right|=0.0010$ with total intensities of $1.30 \times 10^{12}$ and $1.20 \times 10^{12}$ protons in the blue ring and yellow ring, respectively, with 12 bunches per ring. The beam spectra are shown in Fig. 20 (a, blue ring; b, yellow ring) acquired with 1 colliding bunch and 11 non-colliding bunches. The beams were brought into collision by adjusting the relative phase of the radiofrequency (rf) systems between the two accelerators yielding the spectra shown in Fig. 20 (c, blue ring; d, yellow ring) with 1 noncolliding and 11 colliding bunches and $1.22 \times 10^{12}$ and $1.16 \times 10^{12}$ protons in the blue and yellow rings, respectively. Comparing the beam spectra obtained before and after colliding the beams we note:

1) the appearance of strong coherent pi-mode signals in the vertical plane of both beams,

2) strikingly, in the vertical planes a broad range of frequencies between the coherent sigma and pi modes, and

3) a downward shift in the horizontal tune of both beams accompanied by a significant spreading of the tune peaks particularly in the direction of lower betatron tune.

The nature of the observed structure between the observed sigma and pi modes of coherent oscillation is intriguing: while theoretically expected for multiple reasons, to our knowledge the data shown in Fig. 20 are the first ever to evidence this structure. Possible explanations (e.g. multiple modes due to collision schedules, multiple interaction points, long-range interactions, the continuum of incoherent modes, [13]) and further analyses will be presented together with additional measurement results in a later report.

While considerable effort was devoted to improving the resolution of the beam transfer function measurements, the effect of the $10 \mathrm{~Hz}$ tune modulation propagates directly into the measurements of the beam transfer functions and dominates the measured resolution. An example of this is evidenced in the beam spectra shown in Fig. 21 from which is seen what previously was considered a part of "digitizing artifacts" some of which were removed (see section 8). The raw tune data, with 0.003 units full vertical scale, are shown versus time in the inset evidencing significantly more tune modulation at $\sim 10 \mathrm{~Hz}$ in the horizontal (b) versus the vertical (c) plane which is clearly reflected in the measured BTFs.

Shown in Fig. 22 are the raw $\widetilde{\mathbf{I}}$ and $\tilde{\mathrm{Q}}$ signals during the scan of the horizontal beam transfer function from Fig. 21 with an expanded view on the right. These data together with simulation explain the poor signal-tonoise still seen in the measured beam transfer function measurements. Unlike with the multipole correction application (recall Fig. 11), the effects of tune modulation in the beam transfer function application has not yet been filtered out until confidence is gained that all other instrumental anomalies have been addressed.

Between the time of the initial BTF system characterization, shown in Fig. 17 for example, and the data from Fig. 20, several changes (see sections 7 and 8) were made for improved resolution in the BTF measurements. In addition to provision of significantly better tune measurement resolution, the major improvements included:

1) removal of sporadic data randomization due to competing CPU processes resulting in data overwrites,

2) removal of digital filter parameter overwrites due also to competing CPU processes,

3) removal of errors in data synchronization, and

4) implementation of a more precise correction of the frequency-dependent phase response based on network analyzer measurements. 
NON-COLLIDING BEAMS
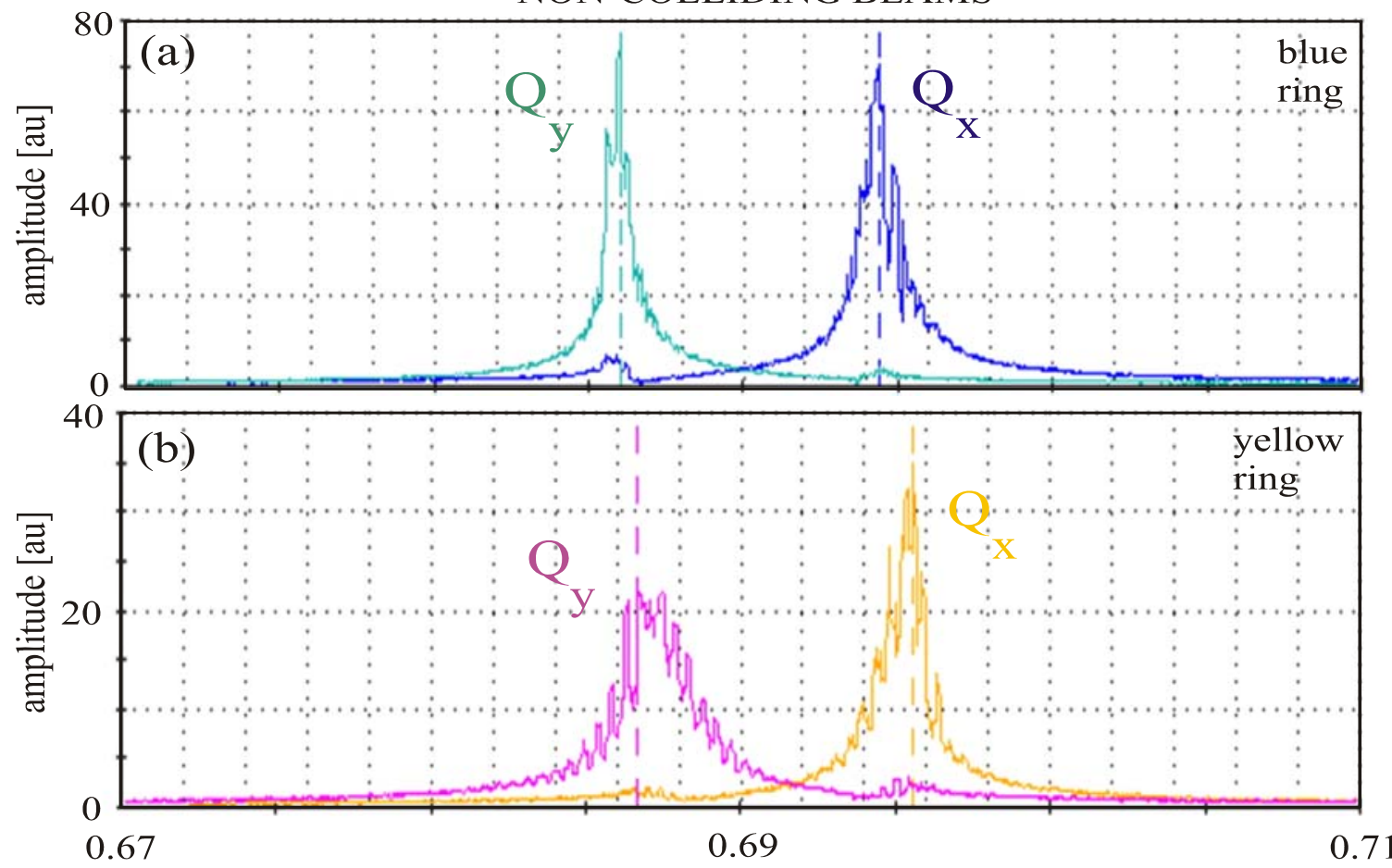

COLLIDING BEAMS
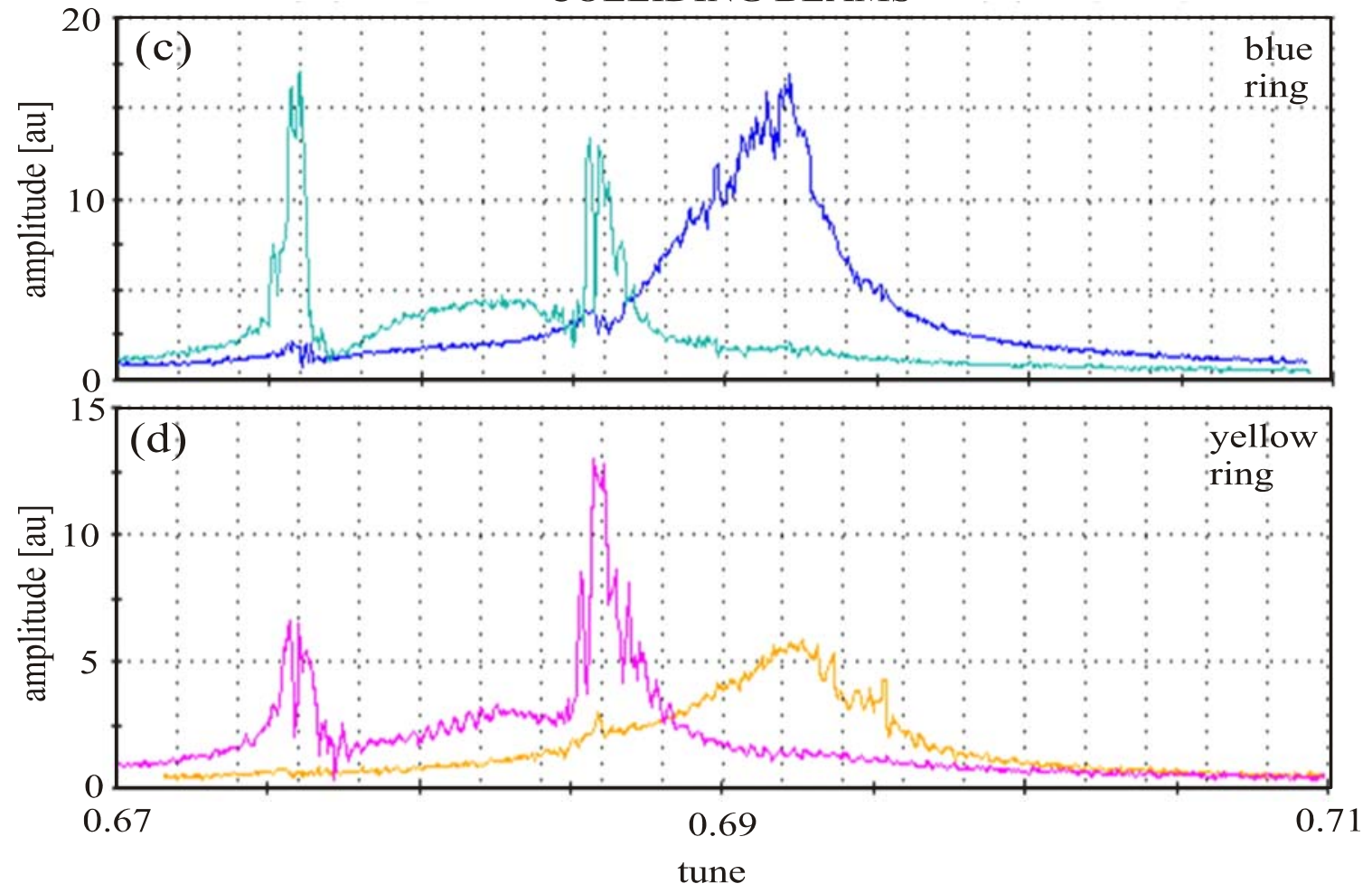

Figure 20: Measured ${ }^{16}$ beam transfer functions with beams in and out of collision evidencing strong coherent beam-beam modes.

${ }^{16}$ Data from 02/26/09, fill 10240. 


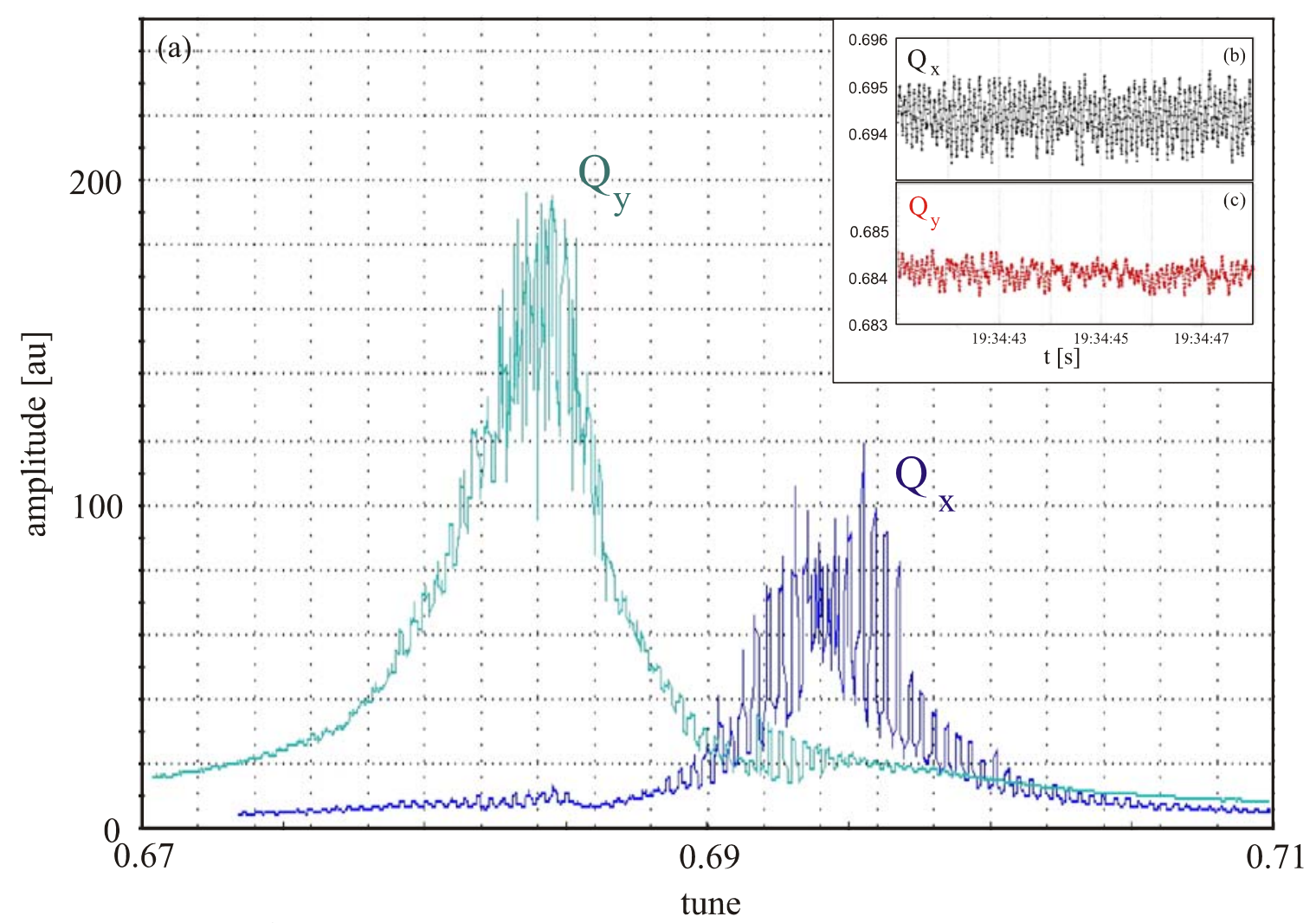

Figure 21: Measured ${ }^{17}$ beam transfer functions in the blue ring (a) in the horizontal and vertical planes and the measured tunes as a function of time in the insets (b, horizontal; c, vertical).
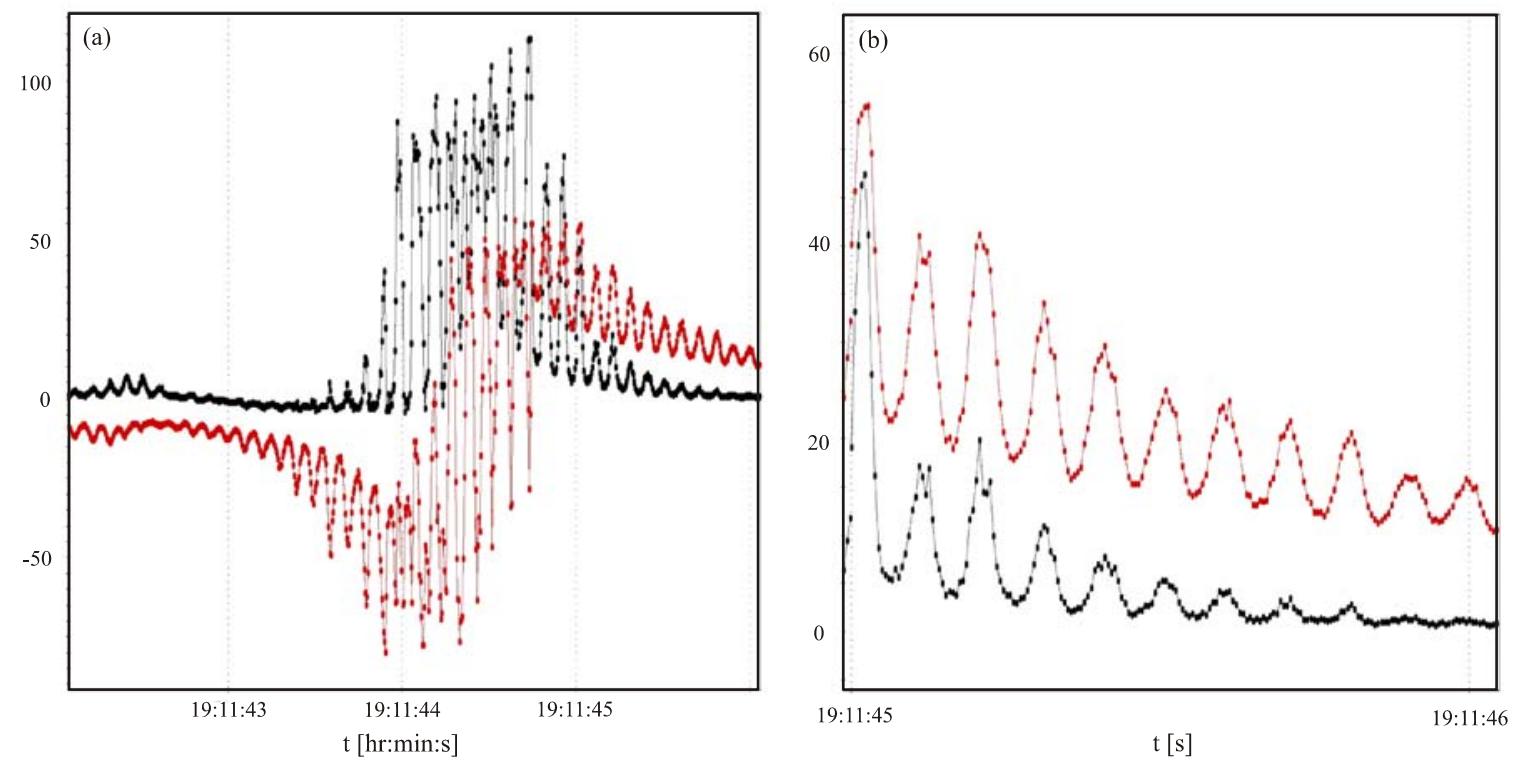

Figure 22: Raw $\tilde{I}$ and $\tilde{Q}$ signals (a) with expanded scale in (b) used as input to the phase lock loop for tune determination during the beam transfer function measurement evidencing strong modulations at a frequency near $10 \mathrm{~Hz}$.

${ }^{17}$ Data from 06/14/09, fill 10928. 


\section{Hardware Improvements}

Nearly fifty issues involving tune measurement hardware items were addressed between December 2008 and July 2009, ranging from replacing damaged cables to modifying chasses to housekeeping. Some of the modifications, additions, and improvements will be described here.

\subsection{Hardware Improvements for Normal Operations}

Among the first major tasks were massive cleanups of the tunnel areas, where the BBQ beamline components were installed, and of the area in Building 1002A where the electronics reside. The tunnel areas contained a great deal of clutter (old cables, connectors, clamps, copper mesh) all of which was picked up, and removed from the tunnel after an activation check. A number of old, unused prototype chassis were still installed in the racks inside 1002A, some with cables still attached; those were also removed. Once the extraneous equipment was eliminated, the remaining equipment was adjusted such that the electronics racks for the blue and yellow rings were identical in layout. Blank panels were added to chasses and racks as needed to cover up empty areas and improve shielding.

The analog front end (AFE) also received many enhancements. Several voltage under-rated capacitors were replaced with properly rated capacitors. Transfer functions of the AFE were simulated and measured, and upon comparison of the results, were found to match well. Furthermore, the two most significant efforts for the AFE focused on adding additional signal test points and modifying components for operation at the near-integer working point.

Prior to adding the additional test points, the internal signal levels of the AFE could not be monitored. The circuit boards were modified to obtain test points for the following signals: +/- input signals from the pickups, the input difference signal, and the filtered signal. The signals can now be observed via two different methods: a Stanford Research Systems Sr785 dynamic signal analyzer (DSA) or a National Instruments Data Acquisition (NI-DAQ or DAQ) system. Two, 2-channel DSA units are installed in Bldg. 1002A, one each for the blue and yellow signals. Typically the difference signals of blue horizontal (BH), blue vertical (BV), yellow horizontal (YH), and yellow vertical (YV) planes are monitored and logged through the controls system, but the signals can be changed locally if necessary. The DSA setup parameters can be controlled locally or remotely. The DAQ system consists of two computers, each with two 8-channel NI PCI-6143 cards that sample at $250 \mathrm{kSamples} / \mathrm{second}$. All four test point signals from each plane (BH, BV, YH, and YV) along with several kicker diagnostic signals are input into peripheral component interconnect (PCI) cards. The DAQ is triggered automatically at the start of every RHIC ramp and collects five minutes of data. The system can also be triggered manually if desired, but it cannot accept new triggers while the DAQ is collecting data. The data is stored in a .tdms format which can be read and analyzed using LabVIEW or MATLAB. Also, an additional driver board was added to each AFE to get the pickup +/- signals into the controls system so a dB-to-mm position calculation would be available. The addition of the test signals also allows the BBQ pickups to be easily centered on the beam rather than the beamline.

As with the AFE, several modifications to obtain test signals from the kicker amplifier were made. First a Hall Effect current transducer was added to each output of the kicker amps, as well as a voltage output to one side of each bridge amplifier. An RMS to DC converter was added to the amplifier current signal, which was input into an MADC for monitoring and logging that signal in the controls system. Additional power supply filtering was added to solve the problem of ripple on both $+/-15 \mathrm{~V}$ supplies. The output of the kicker amplifiers were calibrated to determine the output current for each kicker setting in $\mathrm{dB}$.

Several smaller pieces of the BBQ hardware were also altered for increased reliability. All spare diode boxes were modified to be identical and the end connectors were changed to avoid the use of adaptors. The Khronhite filters and transformers were removed and replaced with passive low pass filters. Mechanical bracing was then added to support the diode boxes and filters. Bracing rods were also attached to the numerically controlled oscillator (NCO) daughter cards so that they make a robust connection and do not flex. Front panels for the NCO modules were machined and mounted. Finally, the NCO outputs were 
connected to a VME 3123 digitizer to allow software to determine the phase of the NCO relative to the ADC sample buffer.

One modulated resonator and a number of fixed-frequency resonators were built years ago to allow the BBQ system to be tested during no-beam conditions. These resonators were unwieldy, resonant frequencies were unable to be ascertained without a test setup, and they were difficult to connect to the system. Therefore, all of the resonators were properly packaged into small boxes with suitable connectors and were labeled with all identifying characteristics. Ultimately, the resonator design was modified slightly to allow for the addition of external capacitors so the resonant frequency could be easily adjusted to match the desired beam optics.

Several improvements were also made to the controls hardware. Screws were added to the MADC connectors to securely seat each connector to the module. Several VME modules were (unnecessarily) replaced during the previous run in an attempt to correct problems with spurious phase jumps in the system (see Appendix B). The modules replaced included the processor, RAM disk, ADC, a V108 board, and a VME controls crate.

With the assistance of the controls group, a duplicate VME was set up in the tech shop to allow for offline testing of new or spare boards, and new software code. Finally, a new Digi was installed in the rack to allow all BBQ FECs (front end computer) to have a serial connection for online diagnostics of each front end.

There are a myriad of interconnects in this system, and signal quality can only be as good as the worst cable. To that end, there was much attention paid to cabling and connectors. The first test performed used a network analyzer to check the integrity of the kicker cables going to the tunnel. Shortly after that, it was noticed that eight feedthroughs on the BBQ pickups needed to be repaired. Extra adaptors were eliminated where possible, and all interconnect points were wrench-tightened. Several broken SMA "T"s were discovered, as well as four $1 / 4$ " Heliax cables that needed to be re-terminated. Where appropriate, cables were re-routed to better paths and were dressed and strain-relieved properly. New cable assemblies were made for both blue and yellow AFE-to-MADC paths as some cables were showing signs of wear (cracking, etc.). Unused blue and yellow MADC inputs were grounded. A dented cable running from the blue $28 \mathrm{MHz}$ output to the splitter box was replaced. Some new cables were run between modules as well to connect the AFE outputs to the opposing plane's ADC, and to use a buffered $28 \mathrm{MHz}$ RF signal from the V124 modules rather than a direct connection to the RF control room.

\subsection{Hardware Developments for Near-Integer Operations}

Several APEX sessions during RHIC Run-9 were devoted to operation with vertical betatron tune near integer. Prior to these sessions, the tunes were expected to be $\mathrm{Q}_{\mathrm{x}}=0.88$ for horizontal and $\mathrm{Q}_{\mathrm{y}}=0.97$ for vertical, yielding (aliased) betatron sidebands of $\mathrm{f}_{\beta \mathrm{x}}=9.4 \mathrm{kHz}$ and $\mathrm{f}_{\beta \mathrm{y}}=2.3 \mathrm{kHz}$ in the horizontal and vertical planes respectively. The filter characteristics of the AFE were such that the experimental horizontal tune would be in the passband of the filter $(-3 \mathrm{~dB}$ low frequency cutoff was around $8 \mathrm{kHz})$, but the vertical sideband would be outside of the passband and therefore attenuated by approximately 20-30 dB. Simulations were run using MATLAB to extend the passband and several components on the AFE were changed to lower the cutoff frequency to around $1 \mathrm{kHz}$. Figure 23 below depicts the measured frequency and phase response of the original AFE response (blue) as compared to the modified AFE response (green).

In summary, the hardware improvements were critical to ensure that the BBQ system would operate robustly throughout the RHIC run. It was necessary to confirm that every piece of hardware functioned as expected and that all interconnects were properly made. Even with the great strides that were made over the course of the past run, the hardware is always undergoing continuous improvement to foster effective operation well into the future. 


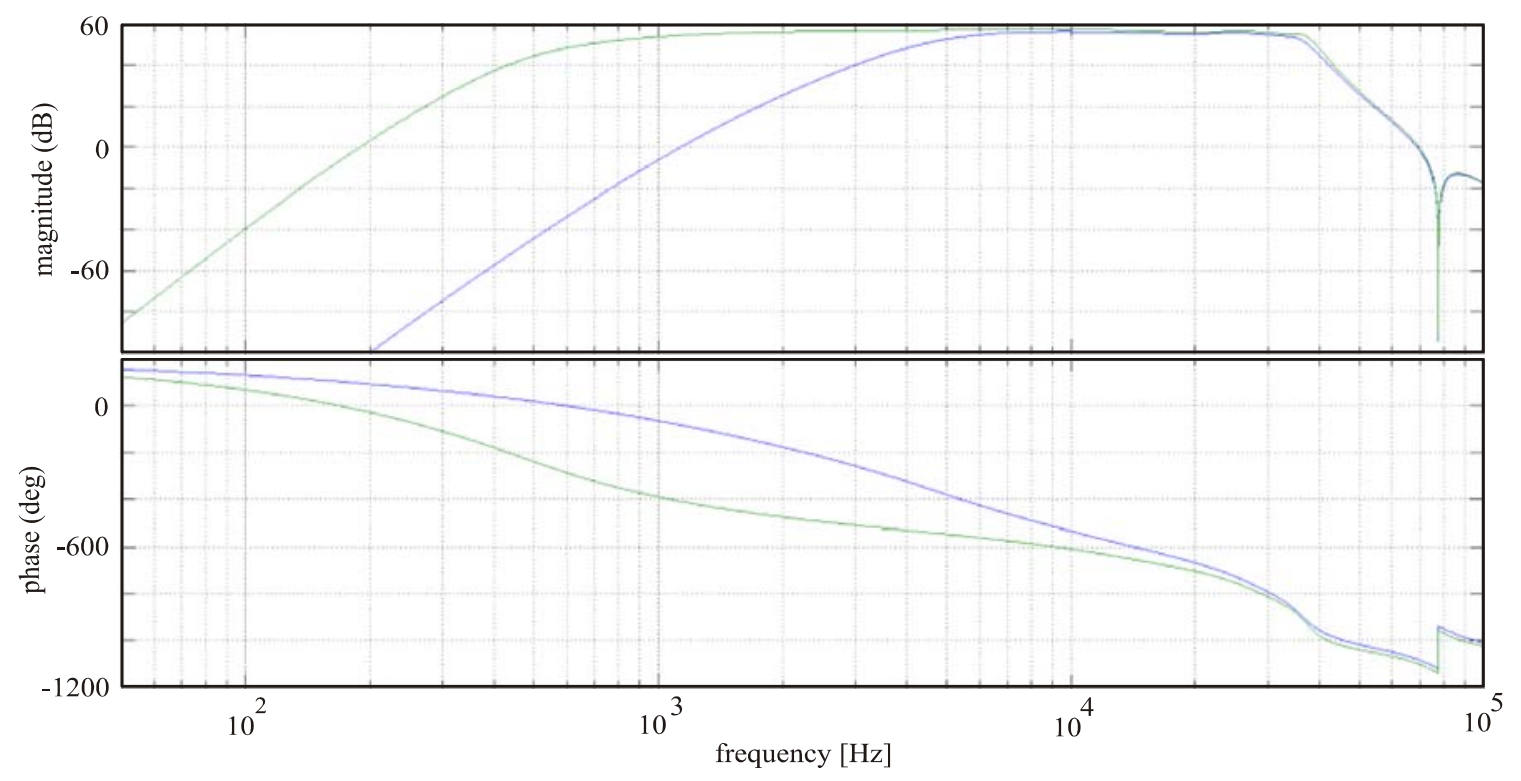

Figure 23: Frequency and phase response of original (blue) and modified (green) analog front end module (AFE). At the ultimately achieved vertical betatron tune of $\mathrm{Q}_{\mathrm{y}}=0.98\left(\mathrm{f}_{\beta \mathrm{y}}=1.6 \mathrm{kHz}\right)$, the signal amplitude was increased by $\sim 35 \mathrm{~dB}$ and the change in phase shift with frequency was increased from $-0.0091 \mathrm{deg} / \mathrm{Hz}$ to $-0.0365 \mathrm{deg} / \mathrm{Hz}$ with the new AFEs.

\section{Software Improvements}

Significantly improved resolution in the measured betatron tunes, the betatron coupling and in the beam transfer function measurements resulted directly from numerous software modifications. The major improvements in this area were developed after realizing that in the limit of fully decoupled beams the signal-to-noise ratio of the coupling measurement approaches unity and that the majority of the data delivered by the tune measurement was being discarded.

\subsection{Software Improvements for Normal Operations}

During previous runs one of the main problems with the BBQ system, from the point of view of tune and coupling feedback, involved noisy tune data and very noisy coupling data. Since the coupling is derived from the amount of signal from the beam in one plane sampled at the betatron frequency of the other plane, the ratio of signal-to-noise in the measurement of betatron coupling is much worse than that for the tune measurement. In the limit of decoupled planes the signal-to-noise ratio for the coupling goes to one; that is, for fully decoupled planes, the measurement of coupling becomes the measurement of noise. The simplest solution for this problem is data averaging. Since the previous algorithm was anyway disregarding 15/16 of the data, signal averaging was easy to implement directly within the existing setup.

From the point of view of signal processing and resulting data rates, the BBQ system operates as follows for the case of fractional tunes of $\sim 0.68$ as used for normal operations:

1. The kicker excites the beam at the betatron frequency $f_{\beta}$ given by the scan frequency $f_{\text {scan }}$ divided by four.

2. The analog front end (AFE) processes the signal.

3. The analog to digital converter (ADC) scans with frequency $\mathrm{f}_{\mathrm{scan}}=\mathrm{f}_{\mathrm{rf}} *(1-\mathrm{Q}) / \mathrm{h} \sim 100 \mathrm{kHz}$, where $\mathrm{f}_{\mathrm{rf}}=28 \mathrm{MHz}$ is the $\mathrm{rf}$ frequency, $\mathrm{h}=360$ is the harmonic number, and $\mathrm{Q}$ is the fractional betatron tune. 
4. The front end computer (FEC) is interrupted every 32 scans $(\sim 3.100 \mathrm{kHz})$, adjusts the scan frequency $\mathrm{f}_{\text {scan }}$ based on the collected data, and delivers data to the magnet control loop on every 16th interrupt at a rate of $\mathrm{f}_{\mathrm{m}} \sim 190 \mathrm{~Hz}$.

5. The magnet control loop sends data to the magnets at this rate $\left(\mathrm{f}_{\mathrm{m}} \sim 190 \mathrm{~Hz}\right)$.

The old code delivered the last sample out of 16 to the magnet control loop while the new code delivers the average of 16 samples, resulting in a significant improvement of data quality (as shown in this document previously).

Other improvements in the data processing were also made:

1. New diagnostic parameters were added to the code and the parameter which shows the number of interrupts processed per second (which should be proportional to the tune) revealed that not all the scans were being processed. This was diagnosed as competition for CPU-time from another process running on the same computer. This process, which was subsequently removed, was previously found to corrupt BTF data. This effect led to random corruption of up to every other tune measurement used with the old, rate-limited, code.

2. The code was also modified to be more deterministic in processing of the data in order to eliminate the possibility of being late to process new data (if the code is not waiting to process new data when the new data become available, the new data will not be processed; e.g. there is no "catch up").

3. For applications requiring just one measurement of the tune per second, instead of providing the last measured point acquired during one second as in the past, the average of all points acquired during that second is delivered, thus further improving data quality (mostly due to averaging out the $10 \mathrm{~Hz}$ modulations present on the beam).

4. During previous runs filters labeled $40 \mathrm{~Hz}$ and $20 \mathrm{~Hz}$ were used. Unfortunately their characteristics were not documented so simulations were performed to determine their response. With an input signal frequency of $25 \mathrm{kHz}$, the full width at half maximum (FWHM) of the passbands of the I/Q demodulator output with no filter, a $40 \mathrm{~Hz}$ filter, and a $20 \mathrm{~Hz}$ filter were found to be respectively $2310 \mathrm{~Hz}, 820 \mathrm{~Hz}$ and $420 \mathrm{~Hz}$. During the run, only " $20 \mathrm{~Hz}$ " filter, although very wide, was used. This filter was specified as a sequence of 11 numbers (i.e. taps), and therefore its characteristics changed in proportion to the scan frequency. That turned out to be very beneficial for near-integer operation, because at lower frequencies the passband of this filter became narrower, for example at $5 \mathrm{kHz}$ the FWHM of the passband is $85 \mathrm{~Hz}$, resulting in substantial reduction of all $60 \mathrm{~Hz}$ harmonics present in the beam signal.

These improvements also greatly benefited the resolution of the beam transfer function (particularly averaging using all delivered tune values). In addition:

1. Frequency changes during the BTF measurement proper were synchronized with the data delivery leading to precise association of data with the frequency those data were taken at.

2. A programming error was found for which digital filter parameters used for one application were overwriting those of another application (causing spikiness in the BTF measurements).

3. Precision phase optimization and frequency-dependent phase correction were implemented.

The magnet control software was also improved in a few important ways. The code which is used to save feedback data (used for subsequent replays) to the networked file system was modified to save cached data and therefore be immune to occasional delays in saving (the old code could only save data from the previous second, which resulted in gaps and corruption of data right after the start of the ramp, presumably due to network slowdown at that time). The code was also modified to stop the magnet control loop from delivering data to magnets when the machine protection systems kicked the beam out of the machine (due to excessive beam losses, magnet failures, etc.) instead of relying on detecting loss of the beam by analyzing tune data. This change prevented quench link interlocks due to sending of unphysical corrections to magnets. 


\subsection{Software Developments for Near-Integer Operations}

Energy ramps with near-integer betatron tunes required further modifications for both the tune measurements and the magnet control software. In the previous encoding, the PID control loop for tunetracking had coefficients independent of the sampling frequency which scales with the betatron frequency and changed by more than a factor of ten when operating near integer. The PID control loop was therefore modified to change these coefficients based on the current data rate in such a way to keep loop characteristics independent of the rate.

The dependence of the numerically controlled oscillator (NCO) phase on NCO frequency was expressed in the previous code as a linear dependence between NCO phase and betatron tune with a hard-wired coefficient. Coincident with the modifications to the AFEs for utilization at near-integer tune, the dependence of NCO phase on NCO frequency was expressed as a linear dependency on NCO frequency with a user-specified linear coefficient.

The most extensive and the most critical modification for near-integer operations concerned the required modification of the magnet control code. With near-integer tunes the data rate for sending tune and coupling corrections to the power supplies was significantly reduced (by a factor of 10 or more). As a result larger requested changes in setpoint were being applied less frequently and the amplitude of the requested corrections exceeded the capabilities of power supplies. To avoid that, the magnet control code was modified to keep the setpoint generation rate above $180 \mathrm{~Hz}$. In other words, tune and coupling corrections are not applied any more, they are ramped to.

\section{Summary and Outlook}

Following the many modifications in hardware and in the data processing algorithms, the time required to establish full-energy beams with the betatron tunes and coupling regulated by feedback was reduced from several weeks to a few hours. Experimental data were presented demonstrating the improved resolution of the tune and coupling measurements which were essential for this achievement. Numerous improvements in the acquisition and processing of data have additionally been implemented for measurements of the beam transfer functions. Hardware (new signal processing modules), software (tune-independent loop parameters, precision frequency-dependent phase compensation), and modifications to power supply control were essential in realizing operation of RHIC at near-integer vertical betatron tunes.

Improved measurement resolution has allowed for detection of beam properties not previously observed including significant externally driven tune and, with textbook clarity, the presence and location of strong beam-beam induced coherent modes as well as significant structure between these modes.

The externally driven tune modulations (and not the resolution) presently limit the achieved precision of the tune and coupling measurements. The tune modulations were shown to have identical frequency content as the measured orbit oscillations indicating a common source. While efforts for eliminating these perturbations to the beam orbit and optics are being pursued with renewed vigor, applications identified to be free of other systematic errors have been modified to input precision average tune measurements.

In the future we anticipate demonstration of chromaticity feedback using tune measurements acquired while modulating the rf frequency during the energy ramp. With the improved measurement resolution a long-standing puzzle concerning chromaticity sign flips observed in previous measurements was unambiguously determined to not result from poor signal-to-noise in the tune measurements. The source has been identified and corrected in the data processing algorithm and prospects appear promising for precision control of the tunes, coupling, and chromaticity in the future.

\section{Acknowledgments}

This work was made possible by the dedicated effort of many individuals over the past several years. Since the initial proof-of-principle with contributing authors given in Reference 1, we would like to thank P. 
Cameron, J. Cupolo, and A. DellaPenna for their instruction during the early part of the shutdown prior to Run-9. For their essential and high quality technical support we (in addition to Tony Curcio and Ron Schroeder) would like to express our sincerest thanks to Craig Rhein, Alan Weston, and Paul Ziminski, who were all involved with the numerous hardware modifications and extremely efficiently and with great attention to detail constructed the new AFEs required for near-integer tune operations. We thank L. Hoff for identifying and correcting the digital filter parameter overwrite problem and for tracking down the spurious query which corrupted system phasing and flipped the sign of the coupling angle. We thank J. Laster for software support of the beam transfer function application. Additionally we thank M. Bai and C. Montag, the $250 \mathrm{GeV}$ and $100 \mathrm{GeV}$ Run-9 coordinators respectively, and both W. Fischer and T. Roser for allowing us the opportunity to independently gain experience with these systems and for many enlightening discussions. Work supported by Brookhaven Science Associates, LLC under Contract No. DE-AC0298CH10886 with the U.S. Department of Energy. 


\section{References}

1. P. Cameron et al., Simultaneous tune and coupling feedback in the Relativistic Heavy Ion Collider, and Possible Implications for the Large Hadron Collider Commissioning, Phys. Rev. ST Accel. Beams 9, 122801 (2006).

2. M. Gasior and R. Jones, The principle and first results of betatron tune measurement by direct diode detection, CERN-LHC-Project-Report 853 (2005), available at http://documents.cern.ch/cgi-bin/setlink?base=preprintl\&cate=cern $\&$ \&id=lhcproj-2005-853.

3. Y. Luo et al., Possible phase loop for the global betatron decoupling, BNL C-AD AP Note 174, (2004).

4. R. Jones et al., Towards a robust phase-locked loop tune feedback system, $7^{\text {th }}$ Eur. Wkshp. on Beam Diagnostics and Instrumentation for Particle Accelerators (DIPAC), Lyon, France (2005).

5. Y. Luo et al., Continuous measurement of global difference coupling using a phase-locked-loop tune meter in the Relativistic Heavy Ion Collider, Phys. Rev. ST Accel. Beams 9, 124001 (2006).

6. P. Cameron et al., Tune, coupling, and chromaticity measurement and feedback during RHIC Run 7\}, $8^{\text {th }}$ Eur. Wkshp. on Beam Diagnostics and Instrumentation for Particle Accelerators (DIPAC), Venice, Italy (2007).

7. A. Della Penna, BBQ StartUp and Run 7, presented at the RHIC Accelerator Physics Experiments (APEX) Workshop (2007), available at http://www.cad.bnl.gov/APEX/APEXWorkshop2007/talks/pm/APEX\%20DellaPenna\%20BBQ3 \%202Nov07.pdf.

8. A. Marusic, Tune and coupling feedback, presented at RHIC Retreat (2007), available at http://www.c-ad.bnl.gov/RHIC/retreat2007/Talks/Marusic\_TF07.pdf.

9. C. Montag et al., Measurements of mechanical triplet vibrations in RHIC, Eur. Part. Accel. Conf. (EPAC) Paris, France (2002).

10. P. Thieberger et al., Active damping tests for a possible cure of the $10 \mathrm{~Hz}$ problem, presented at the RHIC Accelerator Physics Experiments (APEX) Workshop (2008), available at http://www.cad.bnl.gov/APEX/APEXWorkshop2008/talks/Fri_PM_05_Thieberger_APEXActive Dampingtests4.pdf

11. Y. Luo et al., Fast and robust global decoupling with coupling angle modulation, Phys. Rev. ST Accel. Beams 8, 074002 (2005).

12. W. Fischer et al., Transverse beam transfer functions of colliding beams in RHIC, Part. Accel. Conf. (PAC) Albuquerque, NM (2007).

13. W. Herr, Beam-beam interactions, CAS-CERN Accelerator School: Intermediate course on accelerator physics, Zeuthen, Germany, pp. 379-410 (2003), available at http://cdsweb.cern.ch/record/941319/files/p379.pdf. 


\section{Appendix A: Chronology of ramp development with tune/coupling feedback}

The four major modes of accelerator operation using tune/coupling feedback to establish and/or maintain good operating conditions were shown previously in Table 1 . The next tables show for each mode first an overview of the development sessions including date and purpose of the development exercises and then a detail of the time invested to first establish beams to full energy with tune and coupling feedback. Also indicated are the cause for beam loss (if any) and/or the transmission efficiency (for successful ramps).

During run-9, a total of 88 ramps were executed with tune/coupling feedback. Of these, 35 did not result in data suitable for subsequent feed-forward to full beam energies. As summarized previously in Fig. 14, the eight causes for these were identified and are listed below together with frequency of occurrence:

1) uncontrolled orbit excursions resulting in beam loss: [14]

2) human error (fallout from run-8 [1], double-feedforward [2], fallout from APEX testing [1], "newfill" executed during energy ramp [1]): [5]

3) hardware faults, not related to tune/coupling feedback hardware: [4]

4) controls (power supply not tracking setpoint [2], energy and rotator ramp handshake [1]): [3]

5) near-zero chromaticity [3]

6) other (alternate tune "artus"-induced beam loss [1], residual hysteresis [2]): [3]

7) model (estimated setpoint minus measured setpoint exceeded tolerance): [2]

8) tune/coupling feedback hardware (broken SMA tee connector): [1]

\section{Appendix A.1: ramp development summary: $250 \mathrm{GeV} \mathrm{p+p} \mathrm{(02/06/09-04/14/09)}$}

\begin{tabular}{|c|c|c|}
\hline session number & dates & purpose \\
\hline 1 & $02 / 14 / 09-02 / 15 / 09$ & new optic (pp90), blue ring \\
2 & $02 / 15 / 09-02 / 16 / 09$ & new optic (pp90), yellow ring \\
3 & $02 / 17 / 09-02 / 18 / 09$ & new optic (pp91) \\
4 & $02 / 20 / 09$ & support new rf code \\
5 & $02 / 24 / 09$ & patch network latencies \\
6 & $02 / 24 / 09$ & new design tunes \\
7 & $02 / 26 / 09$ & new optic (pp92) \\
8 & $03 / 16 / 09$ & new optic (rot93) \\
9 & $03 / 30 / 09$ & new design tunes \\
10 & $04 / 08 / 09$ & new optic (pp93lowbeta) \\
\hline
\end{tabular}

Table A.1: Summary of ramp development periods used during $250 \mathrm{GeV}, \mathrm{p}+\mathrm{p}$ operation for physics.

Establishing beams to $250 \mathrm{GeV}$ beams was done consecutively in the blue and yellow rings based on accelerator availability (the yellow ring being cooled to liquid Helium temperatures while blue ring commissioning commenced). These efforts are detailed in the next two tables.

\begin{tabular}{|c|c|c|c|c|}
\hline ramp & fill number & date and time & energy $(\mathrm{GeV})$ & beam loss \\
\hline 1 & 10050 & $02 / 14 / 0923: 13$ & 24 & human error \\
2 & 10051 & $02 / 15 / 0900: 24$ & $<154$ & orbit \\
3 & 10052 & $02.15 / 0901: 45$ & $<174$ & model \\
4 & 10053 & $02 / 15 / 0904: 02$ & $<195$ & orbit \\
5 & 10054 & $02 / 15 / 0904: 40$ & $<195$ & orbit \\
6 & 10055 & $02 / 15 / 0905: 02$ & 24 & controls \\
7 & 10056 & $02 / 15 / 0905: 38$ & $<241$ & model \\
8 & 10057 & $02 / 15 / 0906: 16$ & 250 & success \\
\hline
\end{tabular}

Table A.2: Summary of initial ramp development to establish protons at $250 \mathrm{GeV}$ in the blue ring. 


\begin{tabular}{|c|c|c|c|c|c|}
\hline ramp & fill number & date and time & energy $(\mathrm{GeV})$ & beam loss & efficiency \\
\hline \multirow[t]{2}{*}{1} & 10057 & 02/15/09 06:16 & 250 (br) & & $0 \%$ (br) \\
\hline & & & $<37$ (yr) & orbit & $0 \%$ (yr) \\
\hline \multirow[t]{2}{*}{2} & 10058 & 02/15/09 06:56 & 171 (br) & hardware & $0 \%$ (br) \\
\hline & & & 171 (yr) & hardware & $0 \%(\mathrm{yr})$ \\
\hline \multirow[t]{2}{*}{3} & 10081 & 02/16/09 01:13 & 247 (br) & orbit & $0 \%(\mathrm{br})$ \\
\hline & & & 247 (уг) & & $0 \%(\mathrm{yr})$ \\
\hline \multirow[t]{2}{*}{4} & 10082 & 02/16/09 01:51 & 250 (br) & & $72.6 \%$ (br) \\
\hline & & & $250(y r)$ & & $81.8 \%(\mathrm{yr})$ \\
\hline \multirow[t]{2}{*}{5} & 10083 & 02/16/09 02:57 & 250(br) & & $99.6 \%$ (br) \\
\hline & & & $250(y r)$ & & $97.9 \%(\mathrm{yr})$ \\
\hline \multirow[t]{2}{*}{6} & 10084 & 02/16/09 04:04 & 250(br) & & $99.9 \%$ (br) \\
\hline & & & $250(y r)$ & & $94.7 \%(\mathrm{yr})$ \\
\hline \multirow[t]{2}{*}{7} & 10085 & 02/16/0906:12 & 250(br) & & $69.0 \%(\mathrm{br})$ \\
\hline & & & $250(\mathrm{yr})$ & & $97.1 \%(\mathrm{yr})$ \\
\hline
\end{tabular}

Table A.3: Summary of initial ramp development to establish protons at $250 \mathrm{GeV}$ in the blue (br) and yellow (yr) rings.

\section{Appendix A.2: ramp development summary: $100 \mathrm{GeV}$ p+p (04/15/09-06/29/09)}

\begin{tabular}{|c|c|c|}
\hline session number & dates & purpose \\
\hline 1 & $04 / 16 / 09$ & new optic (pp100-90) \\
2 & $04 / 19 / 09$ & new optic (pp90-90rot) \\
3 & $05 / 25 / 09$ & new optic (pp100-91) \\
4 & $06 / 01 / 09-06 / 02-09$ & new optic (pp100-92) \\
5 & $06 / 05 / 09$ & machine development (pp100-90) \\
6 & $06 / 24 / 09$ & machine development (pp100-90) \\
\hline
\end{tabular}

Table A.4: Summary of ramp development periods used during $100 \mathrm{GeV}, \mathrm{p}+\mathrm{p}$ operation for physics.

\begin{tabular}{|c|c|c|c|c|c|}
\hline ramp & fill number & date and time & energy $(\mathrm{GeV})$ & beam loss & efficiency (br/yr) \\
\hline 1 & 10566 & $04 / 16 / 0904: 48$ & 24 & hysteresis & $0 \% / 0 \%$ \\
2 & 10567 & $04 / 16 / 0905: 42$ & 100 & & $23 \% / 36 \%$ \\
3 & 10568 & $04 / 16 / 0906: 54$ & 100 & & $24 \% / 20 \%$ \\
4 & 10569 & $04 / 16 / 0907: 47$ & 100 & & $86 \% / 3 \%$ \\
5 & 10573 & $04 / 16 / 0912: 57$ & 100 & & $86 \% / 12 \%$ \\
6 & 10574 & $04 / 16 / 0913: 54$ & 100 & & $88 \% / 12 \%$ \\
7 & 10575 & $04 / 16 / 0915: 04$ & 100 & & $90 \% / 76 \%$ \\
8 & 10576 & $04 / 16 / 0916: 15$ & 100 & & $89 \% / 76 \%$ \\
9 & 10577 & $04 / 16 / 0916: 59$ & 100 & & $89 \% / 82 \%$ \\
\hline
\end{tabular}

Table A.5: Summary of initial ramp development to establish protons at $100 \mathrm{GeV}$. The latter 5 ramps were executed by the Operations staff. 


\section{Appendix A.3: ramp development summary: $250 \mathrm{GeV}$ p+p, near-integer tune}

\begin{tabular}{|c|c|c|}
\hline session number & dates & purpose \\
\hline 1 & $06 / 10 / 09$ & new optic (pp94nearInt) \\
2 & $06 / 16 / 09-06 / 17 / 09$ & tune optimization \\
3 & $06 / 24 / 09$ & tune optimization \\
4 & $07 / 04 / 09$ & tune optimization \\
\hline
\end{tabular}

Table A.6: Summary of ramp development periods used during $250 \mathrm{GeV}$, p+p operation for accelerator development with near-integer vertical betatron tunes.

\begin{tabular}{|c|c|c|c|c|c|}
\hline ramp & fill number & date and time & energy (GeV) & beam loss & efficiency (br/yr) \\
\hline 1 & 10892 & $06 / 10 / 0917: 48$ & 25 & orbit & $0 \% / 0 \%$ \\
2 & 10893 & $06 / 10 / 0918: 51$ & 27 & orbit & $0 \% / 0 \%$ \\
3 & 10894 & $06 / 10 / 0919: 41$ & 250 & & $67 \% / 19 \%$ \\
4 & 10895 & $06 / 10 / 0921: 08$ & 249 & chromaticity & $0 \% / 0 \%$ \\
5 & 10896 & $06 / 10 / 0922: 25$ & 250 & hardware & $0 \% / 0 \%$ \\
6 & 10897 & $06 / 10 / 0923: 14$ & 250 & & $89 \% / 54 \%$ \\
7 & 10898 & $06 / 10 / 0923: 48$ & 223 & chromaticity & $0 \% / 0 \%$ \\
\hline
\end{tabular}

Table A.7: Summary of initial ramp development to establish protons at $250 \mathrm{GeV}$ with near-integer vertical betatron tunes.

\section{Appendix A.4: ramp development summary: $100 \mathrm{GeV}$ p+p, pp2pp experiment $(06 / 29 / 09-07 / 04 / 09)$}

\begin{tabular}{|c|c|c|}
\hline session number & dates & purpose \\
\hline 1 & $06 / 29 / 09$ & new optic (pp100-90pp2pp) \\
\hline
\end{tabular}

Table A.8: Summary of ramp development period used during $100 \mathrm{GeV}, \mathrm{p}^{+} \mathrm{p}$ operation for pp2pp experiment.

\begin{tabular}{|c|c|c|c|c|c|}
\hline ramp & fill number & date and time & energy (GeV) & beam loss & efficiency (br/yr) \\
\hline 1 & 11008 & $06 / 29 / 0912: 20$ & 100 & orbit & $0 \% / 0 \%$ \\
2 & 11009 & $06 / 29 / 0913: 05$ & 100 & chromaticity & $99 \% / 0 \%$ \\
3 & 11010 & $06 / 29 / 0913: 54$ & 100 & & $100 \% / 92 \%$ \\
4 & 11011 & $06 / 29 / 0915: 02$ & 100 & & $100 \% / 97 \%$ \\
5 & 11012 & $06 / 29 / 0916: 13$ & 100 & & $100 \% / 99 \%$ \\
6 & 11013 & $06 / 29 / 0918: 23$ & 100 & & $100 \% / 99 \%$ \\
\hline
\end{tabular}

Table A.9: Summary of initial ramp development to establish protons at $100 \mathrm{GeV}$ for pp2pp experiment. 


\section{Appendix B: Post Run Performance Analysis}

An outstanding problem related to ambiguities in the sign of the applied coupling correction was diagnosed shortly before the start of run-9. This was related to phase jumps (modulo $\frac{\pi}{2}$ times integer) between the frequency reference from the numerically controlled oscillator and the I/Q demodulator. These were found to be caused by an automatic logging query of an unused parameter in an accelerator device object (ADO) used in signal processing and was eliminated by removal of the offending parameter.

Previous experiences with strong signal contributions at multiples of $60 \mathrm{~Hz}$ contaminating the measured frequency spectrum of the beam [1,5-7], while initially absent but observed to increase in amplitude throughout the course of run-9, did not pose a problem as in the past. Operation with "tune swing" (as used with operation at $100 \mathrm{GeV}$ ) for which the design tunes vary by $\sim 0.05$ encompassing $\sim 65$ multiples of $60 \mathrm{~Hz}$ was uneventfully successful. Further remediation measures have been developed (but not yet implemented) in the event that the signal strength of these harmonics becomes intolerable.

As noted in earlier years, the cycling of the Booster accelerator magnets introduces spikes in the recorded data. For this reason, the Booster ramp is deactivated (for $\sim 5$ minutes) for energy ramps requiring high precision tune and coupling feedback.

For the majority of run-9, tune/coupling feedback operated reliably without hardware or software failures. A problem noted (during high-intensity beam operation, or "scrubbing" for removal of electron-cloud sources), concerning phase jumps in the blue ring during injection of the yellow ring, while having no impact on tune/coupling feedback, remains unsolved. During the last $\sim 3$ weeks however, the system was fraught with a plethora of errors manifesting themselves in many ways:

1) phase jumps occurring in all 4 planes with intermittent, uncorrelated frequency

2) a problem with time-stamping of the diagnostic data

3) missing data observed first while testing new chromaticity feedback in all 4 planes

4) missing data in various underlying logged parameters in all 4 planes

5) data corruption in the BTF application in all 4 planes

Much activity focused around diagnosis of item 1 including online installation of in-house V124 modules to shield against dropouts of the rf frequency, multiple (unnecessary) VME module swaps, online installation of a real-time phase jump compensator (to be utilized in the future only after maintenance days during which time the systems are prone to component reboots), offline testing of ADC and FEC limitations, offline testing of the benefits of higher-speed processors, etc. The cause has recently been diagnosed and verified to have been caused by improper grounding of the digitizer inputs (model VME3123) causing the ADC to miss samples.

Item 2 above was diagnosed and has been resolved.

Items 3-5, while at the time not obviously linked to a common source, have also been diagnosed. While item 3 was initially thought to be due to CPU limitations during the CPU-intensive computation of chromaticities, the problem was found to persist after backing out the new code. Missing data and data corruption (while not time-correlated, rather random in nature) were found to be due to a logging failure which resulted from a failed network switch. The failure of the control system to detect the problem and the susceptibility to human error (error overrides) in these critical systems are a cause of concern.

Lastly, issues related to network latency (recall Table A.1), or missing data during the first $\sim 1 \mathrm{~s}$ of the energy ramp, were recently diagnosed and corrected. While suspected to result from network bandwidth limitations, in fact the cause was rather an unfortunate serial (as opposed to parallel) connection of two network switches combined with a mixing of front-end computers (FECs) and peripheral hardware (computers and scopes) on these switches. A consequence of the old topology was that scope queries and/or computer usage would override system critical tasks (such as commands issued by the magnet manager). 